\title{
A FINITE ELEMENT SCHEME FOR THE EVOLUTION OF ORIENTATIONAL ORDER IN FLUID MEMBRANES
}

\author{
Sören Bartels ${ }^{1}$, Georg Dolzmann ${ }^{2}$ and Ricardo H. Nochetto ${ }^{3}$
}

\begin{abstract}
We investigate the evolution of an almost flat membrane driven by competition of the homogeneous, Frank, and bending energies as well as the coupling of the local order of the constituent molecules of the membrane to its curvature. We propose an alternative to the model in [J.B. Fournier and P. Galatoa, J. Phys. II 7 (1997) 1509-1520; N. Uchida, Phys. Rev. E 66 (2002) 040902] which replaces a Ginzburg-Landau penalization for the length of the order parameter by a rigid constraint. We introduce a fully discrete scheme, consisting of piecewise linear finite elements, show that it is unconditionally stable for a large range of the elastic moduli in the model, and prove its convergence (up to subsequences) thereby proving the existence of a weak solution to the continuous model. Numerical simulations are included that examine typical model situations, confirm our theory, and explore numerical predictions beyond that theory.
\end{abstract}

Mathematics Subject Classification. 35K55, 74K15.

Received November 27, 2008. Revised June 11, 2009.

Published online October 9, 2009.

\section{INTRODUCTION}

Lipid bilayers are the fundamental building block for fluid membranes that are the main constituent of cell membranes in almost all cells of living organisms. Their formation is driven by the tendency to shield the hydrophobic tails of the amphiphilic lipid molecules from the aqueous environment. From a mathematical point of view, the first papers $[10,18,24,26]$ were inspired by the quest to understand the biconvex shape of red blood cells based on an energy minimization principle (see also the review [37]).

Most models investigated so far are typically based on the assumptions (see, e.g., [10]) that the membrane consists of two isotropic labile surfaces, that there is no exchange of molecules between the two sides, and that the membrane has the same physical properties over the entire surface. The conformation of the membrane is driven by the curvature energy from bending elasticity, additional geometric constraints like fixed enclosed volume or surface area, and a signature of the bilayer aspect of the membrane. More recent work explores the formation and dynamics of co-existing phases or domains on the membrane separated by line tension [6], as well as the fluid-membrane coupling [12].

\footnotetext{
Keywords and phrases. Biomembrane, orientational order, curvature.

1 Universität Bonn, Institut für Numerische Simulation, Wegelerstr. 6, 53115 Bonn, Germany.

2 NWF I - Mathematik, Universität Regensburg, 93040 Regensburg, Germany.

3 Mathematics Department and Institute for Physical Science and Technology, University of Maryland, College Park, MD 20742, USA.
} 
In this paper we focus on the analysis of mathematical models for membranes in the gel phase in which the thermal fluctuations of the hydrophobic tails are so small that one can define a two-dimensional order parameter or director. It is experimentally observed that the tails form a fixed angle with respect to the layer normal and have locally the same orientation [31,32]. The interfaces between the regions of different orientation, or domains, and their dynamics are of particular interest in artificial vesicles since they could be used as functional parts of the membrane.

In order to formulate the energy of the system we adopt the local description in the Monge Gauge, that has been widely used in the literature. We follow the classical references $[29,30,38]$ by defining the two-dimensional director $n$ as the projection of the direction of the tails onto the tangent plane to the surface and we define the symmetric and traceless order parameter $Q$ by

$$
Q=S\left(n \otimes n-\frac{1}{2} I_{2 \times 2}\right)
$$

where $S$ is a variable scalar parameter. We assume that the energy of a flat piece of the membrane, described by a graph $u$ over a two-dimensional domain $\Omega$, is zero while a curved membrane has stored elastic energy. In the linearized setting proposed by Uchida [41], corresponding to an almost flat membrane $|\nabla u| \ll 1$ with periodic boundary conditions (see also [33] and the references therein), the energy $E$ of the system

$$
E[u, Q]=\int_{\Omega}\left(f_{\mathrm{hom}}[Q]+f_{F}[Q]+f_{\operatorname{curv}}[u, Q]\right)
$$

consists of three parts, the homogeneous, Frank, and curvature-elastic terms

$$
\begin{aligned}
f_{\text {hom }}[Q] & =-\frac{\alpha}{2}|Q|^{2}+\frac{\beta}{4}|Q|^{4}, \\
f_{F}[Q] & =\frac{\xi}{2}|\nabla Q|^{2}, \\
f_{\text {curv }}[u, Q] & =\frac{\kappa}{2}(\Delta u)^{2}+\delta Q: D^{2} u,
\end{aligned}
$$

where $|Q|^{2}=\sum_{i, j} Q_{i j}^{2}$ is the square of the Frobenius norm of the matrix $Q, \Delta u$ is the Laplacian of the height $u$ (linearized mean curvature), and $D^{2} u$ is the Hessian of $u$; moreover, $\alpha, \beta, \xi, \kappa, \delta>0$ are parameters of the model. The coupling between the elastic deformation and the order parameter is a lowest order approximation of the form $Q: D^{2} u=\sum_{i, j, k} E_{i j} Q_{j k} \partial_{i} \partial_{k} u$, where $E_{i j}=\delta_{i j}$ is the unit tensor for an achiral membrane [19] or the totally antisymmetric tensor for a chiral membrane [34]; hereafter we consider the achiral case as in [41]. This is for example true for dioleoyl phosphatidylcholine (DOPC) which is frequently used in artificial membranes.

The numerical analysis of models related to the shape of fluid membranes is a challenging problem due to the complex interplay of several important physical mechanisms. Previous work has mainly focused on the phase field approach for the Canham-Helfrich functional $[15,16]$, which assumes that the shape of the vesicle is determined from minimizing the square of mean curvature, possibly taking into account the phenomenon of spontaneous curvature [24,37]. The corresponding gradient flow dynamics are usually referred to as Willmore flow [43], and pose a challenging mathematical problem $[4,14,17,36]$. The combination of a phase field model also incorporating hydrodynamical constraints has been pursued in [7]. The membrane-fluid interaction has been recently investigated theoretically [12] as well as numerically [35].

We present a model that allows for effective computations with a minimal number of free parameters. Therefore we replace the homogeneous energy $f_{\text {hom }}[Q]$, which contains two parameters, with the constraint $|Q|^{2}=1 / 2$. This is justified in Section 2.1 as a formal limit of a Ginzburg-Landau energy when the penalization parameter tends to zero and, in turn, implies that the scalar parameter $S$ in (1.1) satisfies $S \rightarrow 1$ in the limit. Such a constraint is natural in many systems with orientational order. For example, the simplest models for liquid crystals reduce to a minimization of the Dirichlet energy subject to this constraint [42]. We regard our model, 
and the ensuing discrete method, as a first step in estimating the coupling parameters $\xi, \kappa$, and $\delta$ based on a comparison of experiment and numerical simulation. The full model on a closed surface should penalize the condition that the orientation of the tails of the lipid molecules has a fixed angle with respect to the tangential plane to the membrane surface since there are no smooth vector fields tangential to a closed surface in $\mathbb{R}^{3}$.

We introduce a fully discrete finite element scheme which is practical. Our numerical method is flexible in terms of boundary conditions, and is capable of simulating the dynamics over coarse meshes. This is due to the fact that we avoid the diffusion mechanism typical of Ginzburg-Landau models which include a small parameter $\epsilon$. These models allow (regularized) defects of degree $s$, namely singular solutions of the form $n(x) \approx \exp (\mathrm{i} \arg (x))$ with $s= \pm 1 / 2, \pm 1$ and whose energy is logarithmic in $\epsilon$. Singularities of this form have infinite energy in the constrained model and are therefore not admissible. However, our discrete model introduces an alternate finite length scale, the meshsize $h$, and thus admits a discrete version of these defects. We discuss this issue in Section 6.

This paper is the first to present a rigorous mathematical analysis of a model that couples the orientation of lipid molecules with membrane bending. The gradient flow dynamics of the free energy gives rise to a coupled parabolic system. In Section 4, we find the range for the coupling parameter $\delta$ within which the discrete system is unconditionally stable. This leads to several a priori bounds. We then prove in Section 5 that the discrete solutions converge, thereby showing existence of a weak solution for the limit system. We conclude with several numerical simulations in Section 6; some of them examine typical model situations within theory while others explore numerical predictions for defects beyond theory.

\section{Mathematical MOdel AND WEAK FORMUlation}

We now modify Uchida's model [41] in several respects and present a weak formulation and discretization, along with the main theoretical results of this paper; the model in [41] may be viewed as a simplified version of that in [19]. We show first via nondimensionalization that the homogeneous energy $f_{\text {hom }}[Q]$ is a penalization term for $|Q|$ and replace it by a rigid constraint. We next change the energy to accommodate boundary conditions other than periodic. We finally present the discrete scheme and corresponding numerical analysis.

\subsection{Nondimensionalization}

Let $L>0$ be comparable to the diameter of the domain $\Omega$, and set $\varepsilon=L^{-1}$. If $\gamma=\sqrt{\beta} / \sqrt{2 \alpha}$, we impose the change of variables $\hat{x}=\varepsilon x$ and

$$
\hat{u}(\hat{x})=\varepsilon u(x), \quad \hat{Q}(\hat{x})=\gamma Q(x), \quad \forall x \in \Omega
$$

We observe that this scaling preserves the slope of membrane height, namely $\hat{\nabla} \hat{u}=\nabla u \ll 1$, which is a critical assumption to formulate the linearized model (1.2). The rescaled energy $\hat{E}$ is given by

$$
\hat{E}[\hat{u}, \hat{Q}]=\int_{\hat{\Omega}}\left\{\frac{\alpha^{2}}{2 \varepsilon^{2} \beta}|\hat{Q}|^{2}\left(|\hat{Q}|^{2}-1\right)+\frac{\xi}{2 \gamma^{2}}|\hat{\nabla} \hat{Q}|^{2}+\frac{\kappa}{2}|\hat{\Delta} \hat{u}|^{2}+\frac{\delta}{\gamma \varepsilon} \hat{D}^{2} \hat{u}: \hat{Q}\right\} \mathrm{d} x
$$

The parameters reported in (1.2) are

$$
\alpha=1, \quad \beta=20, \quad \xi=\delta=1, \quad \kappa=20, \quad L=512,
$$

which lead to $\frac{\alpha^{2}}{2 \varepsilon^{2} \beta} \approx 0.65 \times 10^{4}$. We can thus regard the homogeneous term as a Ginzburg-Landau penalization of the rigid constraint $|\hat{Q}|=1 / \sqrt{2}$. 
Therefore, from now on we relabel all functions and parameters involved, impose the rigid constraint $|Q|=1 / \sqrt{2}$, and rewrite the rescaled energy in (2.2) as follows:

$$
E[u, Q]=\int_{\Omega}\left\{\frac{\xi}{2}|\nabla Q|^{2}+\frac{\kappa}{2}|\Delta u|^{2}+\delta D^{2} u: Q\right\} \mathrm{d} x
$$

\subsection{Modified energy and Euler-Lagrange equations}

In Uchida's model the domain $\Omega$ is a square and periodic boundary conditions are imposed for both $u$ and $Q$. Since this does not provide enough flexibility for the study of the coupled system, we further modify the energy $E$ to accommodate the boundary conditions:

$$
u=u_{D}, \quad \Delta u=g \quad \text { on } \Gamma .
$$

For $g=0$ this corresponds to a freely supported Kirchhoff plate. We claim that the correct form of the energy $E[u, Q]$ in $(2.4)$ is now

$$
E[u, Q]=\int_{\Omega}\left\{\frac{\xi}{2}|\nabla Q|^{2}+\frac{\kappa}{2}|\Delta u|^{2}-\delta \nabla u \cdot \operatorname{Div} Q\right\} \mathrm{d} x-\kappa \int_{\Gamma} g \partial_{\nu} u \mathrm{~d} S,
$$

where $\operatorname{Div} Q$ is a column vector obtained by computing the divergence of each row of the matrix $Q$. To see this we compute formally the variational derivative $\delta E / \delta u$ with respect to $u$ :

$$
\left\langle\frac{\delta E}{\delta u}, v\right\rangle=\int_{\Omega}\{\kappa \Delta u \Delta v-\delta \nabla v \cdot \operatorname{Div} Q\} \mathrm{d} x-\kappa \int_{\Gamma} g \partial_{\nu} v \mathrm{~d} S
$$

where the variation $v$ vanishes on $\Gamma$. Formal integration by parts yields

$$
\begin{aligned}
\left\langle\frac{\delta E}{\delta u}, v\right\rangle & =\int_{\Omega}\{-\kappa \nabla \Delta u \cdot \nabla v+\delta \operatorname{div} \operatorname{Div} Q v\} \mathrm{d} x+\kappa \int_{\Gamma}(\Delta u-g) \partial_{\nu} v \mathrm{~d} S \\
& =\int_{\Omega}\left(\kappa \Delta^{2} u+\delta \operatorname{div} \operatorname{Div} Q\right) v \mathrm{~d} x+\kappa \int_{\Gamma}(\Delta u-g) \partial_{\nu} v \mathrm{~d} S .
\end{aligned}
$$

This reveals the Euler-Lagrange equation for $u$, namely,

$$
\frac{\delta E}{\delta u}=\kappa \Delta^{2} u+\delta \operatorname{div} \operatorname{Div} Q=0,
$$

as well as the desired boundary condition (2.5). We observe that (2.6) is consistent with (1.2) for periodic boundary conditions because integration by parts gives

$$
-\int_{\Omega} \nabla u \cdot \operatorname{Div} Q \mathrm{~d} x=\int_{\Omega} D^{2} u: Q \mathrm{~d} x
$$

without boundary contributions. Consequently, we deal with (2.6) from now on.

We can yet simplify matters a bit further upon realizing that a symmetric and traceless matrix $Q$ can be written as follows:

$$
Q=A q=\frac{1}{2}\left[\begin{array}{rr}
q_{1} & q_{2} \\
q_{2} & -q_{1}
\end{array}\right],
$$


where $A$ is a linear operator. Moreover, imposing the constraint $|Q|=1 / \sqrt{2}$ is equivalent to the holonomic constraint $|q|=1$ for $q=\left(q_{1}, q_{2}\right)$. To derive the Euler-Lagrange equation for $q$ we construct first a Lagrangian, or augmented energy, to account for the constraint $|q|=1$, namely,

$$
L[u, q, \lambda]=\int_{\Omega}\left\{\frac{\mu}{2}|\nabla q|^{2}+\frac{\kappa}{2}|\Delta u|^{2}-\delta \nabla u \cdot \operatorname{Div} A q+\frac{\lambda}{2}\left(|q|^{2}-1\right)\right\} \mathrm{d} x-\kappa \int_{\Gamma} g \partial_{\nu} u \mathrm{~d} S,
$$

where we write $\mu=2 \xi$. Formal variational differentiation of $L[u, q, \lambda]$ with respect to $q$ implies

$$
\left\langle\frac{\delta L}{\delta q}, p\right\rangle=\int_{\Omega}\{\mu \nabla q \cdot \nabla p-\delta \nabla u \cdot \operatorname{Div} A p+\lambda q \cdot p\} \mathrm{d} x=0
$$

where the variation $p$ vanishes on $\Gamma$. Formal integration by parts yields

$$
\frac{\delta L}{\delta q}=-\mu \Delta q+\delta A^{*} D^{2} u+\lambda q=0,
$$

where $A^{*}$ is the adjoint of $A$ and reads $A^{*} D^{2} u=\left[\partial_{1}^{2} u-\partial_{2}^{2} u, 2 \partial_{12} u\right]^{T}$. To find the multiplier $\lambda$ we multiply the equation above by $q$ and use that $|q|=1$ :

$$
\lambda=\mu q \cdot \Delta q-\delta D^{2} u: A q=-\mu|\nabla q|^{2}-\delta D^{2} u: A q .
$$

In fact, for all $v \in C_{0}^{\infty}(\Omega)$ and all $j$, we can write

$$
\int_{\Omega} v \partial_{j}^{2} q \cdot q \mathrm{~d} x=-\int_{\Omega} \partial_{j} q \cdot \partial_{j} q v \mathrm{~d} x-\int_{\Omega} \partial_{j} q \cdot q \partial_{j} v \mathrm{~d} x=-\int_{\Omega} \partial_{j} q \cdot \partial_{j} q v \mathrm{~d} x
$$

because $\partial_{j} q \cdot q=0$. This amounts to $q \cdot \Delta q=-|\nabla q|^{2}$ and gives rise to the Euler-Lagrange equation for $q$

$$
-\mu \Delta q+\delta A^{*} D^{2} u-\left(\mu|\nabla q|^{2}+\delta A^{*} D^{2} u \cdot q\right) q=0 .
$$

A different approach to the elasticity of membranes based on Cosserat surface theory has been pursued in the literature; see e.g. [39] and the references therein. However, it seems that the analogue of our model situation with a fixed angle between the molecules and the layer normal has not yet been investigated in this context.

\subsection{Special solutions and asymptotics}

To get some insight on the interplay between geometry and director field, we seek minimizers of $E[u, q]=$ $L[u, q, \lambda]$ given by $(2.9)$ for $|q|=1$. We drastically simplify the search by considering quadratic functions $u$ of the form

$$
u\left(x_{1}, x_{2}\right)=\sigma x_{1}^{2}+x_{2}^{2},
$$

and a constant vector field $q$. Invoking $(2.5)$ and $\Delta u=2(\sigma+1)$, we see that

$$
L[u, q, \lambda]=\frac{\kappa}{2} \int_{\Omega}|\Delta u|^{2} \mathrm{~d} x-\kappa \int_{\Gamma} g \partial_{\nu} u \mathrm{~d} S=-\frac{\kappa}{2} \int_{\Omega}|\Delta u|^{2} \mathrm{~d} x=-2 \kappa(\sigma+1)^{2}|\Omega|
$$

does not depend on the value of $q$. To extract a nontrivial relation between $u$ and $q$ we resort to (2.10), which gives the equations

$$
\begin{aligned}
\left(\partial_{1}^{2} u-\partial_{2}^{2} u\right)\left(1-q_{1}^{2}\right) & =2 \partial_{12}^{2} u q_{1} q_{2}, \\
\left(\partial_{1}^{2} u-\partial_{2}^{2} u\right) q_{1} q_{2} & =2 \partial_{12}^{2} u q_{1} q_{2} .
\end{aligned}
$$


In view of (2.11), this translates into the equations

$$
2(\sigma-1)\left(1-q_{1}^{2}\right)=2(\sigma-1) q_{1} q_{2}=0 .
$$

We now choose $\sigma \neq 1$, which implies that the principal curvature directions of the membrane described by $u$ are parallel to the coordinates axes and

$$
q_{1}= \pm 1, \quad q_{2}=0 .
$$

Combining (1.1), with $S=1$, and (2.8) we obtain the following relations between the director field $n$ and $q$ :

$$
n_{1}^{2}=\frac{1}{2}\left(1+q_{1}\right), \quad n_{1} n_{2}=\frac{1}{2} q_{2},
$$

whence

$$
q_{1}=1 \quad \Rightarrow \quad\left|n_{1}\right|=1, n_{2}=0 ; \quad q_{1}=-1 \quad \Rightarrow \quad n_{1}=0,\left|n_{2}\right|=1 .
$$

Consequently we conclude that

The director field $n$ aligns with one of the directions of principal curvature of the membrane, and the energy of an ellipsoid $(\sigma>0)$ is smaller than that of a saddle $(\sigma<0)$.

This elementary calculation does not show any preferred curvature direction, which seems to be consistent with interchanging the upper and the lower layer of the membrane. We point out that a model that is quadratic in the coupling expression $D^{2} u: Q$ was proposed in [8] and promotes the alignment of $n$ with a zero curvature direction.

The study of simple membranes of the form (2.11) sheds some light on equilibrium configurations, which are usually a combination of local bumps and saddles (see Sect. 6); they can be reached as asymptotics of the dynamics described below. Note also that they do not satisfy periodic boundary conditions and, therefore, cannot be special solutions of Uchida's model [41].

\subsection{Dynamics}

Following Uchida [41], we consider an evolution defined through the $L^{2}$-gradient flow of $L$, i.e., through the parabolic, nonlinear, coupled system of partial differential equations

$$
\begin{aligned}
& \partial_{t} q=-\gamma_{q} \frac{\delta L}{\delta q}=-\gamma_{q}\left(-\mu \Delta q+\delta A^{*} D^{2} u+\lambda q\right), \\
& \partial_{t} u=-\gamma_{u} \frac{\delta L}{\delta u}=-\gamma_{u}\left(\kappa \Delta^{2} u+\delta \operatorname{div} \operatorname{Div} A q\right),
\end{aligned}
$$

as results from (2.7) and (2.10); here $\lambda=-\mu|\nabla q|^{2}-\delta D^{2} u: A q$ is the Lagrange multiplier that corresponds to the constraint $|q|=1$ almost everywhere.

We supplement the problem with initial conditions

$$
q(0, \cdot)=q_{0} \quad \text { and } \quad u(0, \cdot)=u_{0}
$$

for given $q_{0} \in H^{1}\left(\Omega ; \mathbb{R}^{2}\right)$ satisfying $\left|q_{0}\right|=1$ almost everywhere in $\Omega$ and $u_{0} \in H^{3}(\Omega)$ as well as compatible, time-independent boundary conditions for $t>0$

$$
\begin{aligned}
\left.q(t, \cdot)\right|_{\Gamma} & =q_{\mathrm{D}}=\left.q_{0}\right|_{\Gamma}, \\
\left.u(t, \cdot)\right|_{\Gamma} & =u_{\mathrm{D}}=\left.u_{0}\right|_{\Gamma}, \\
\left.\Delta u(t, \cdot)\right|_{\Gamma} & =g=\left.\Delta u_{0}\right|_{\Gamma} .
\end{aligned}
$$




\subsection{Weak formulation}

In order to state precise mathematical results for the Lagrangian (2.9) we need the space regularity $u \in H^{2}(\Omega)$ and $Q \in H^{1}\left(\Omega ; \mathbb{R}^{2 \times 2}\right)$. Since our analysis requires full $H^{2}$ regularity for the Laplace operator, we hereafter assume that $\Omega$ is a convex and polygonal domain. This is not a serious restriction in this setting since $\Omega$ is typically a square; see [41] and the simulations of Section 6.

The boundary condition $\Delta u=g$ corresponds to the case of a freely supported plate. They are the natural boundary conditions in our variational formulation and therefore included in the energy functional and not in the function spaces, as already discussed in Section 2.2. As usual, we may replace $u$ by $u+u_{0}$ in order to reduce the analysis of the system to the case of zero boundary conditions for $u$. Moreover, the boundary integral in (2.9) does not pose any additional difficulties and, hence, we assume in the following that

$$
\left.u(t, \cdot)\right|_{\Gamma}=u_{\mathrm{D}}=0,\left.\quad \Delta u(t, \cdot)\right|_{\Gamma}=g=0
$$

for $t>0$. Alternatively we could impose periodic boundary conditions, for which the boundary terms cancel by periodicity.

Notation. We use the standard notation for the function spaces of vector-valued functions and we let $(\cdot, \cdot)_{X}$ denote the inner product in $L^{2}\left(X ; \mathbb{R}^{\ell}\right), \ell \geq 1$. We omit $X$ whenever $\Omega$ is meant. Moreover, we set $\|\cdot\|=(\cdot, \cdot)^{1 / 2}$.

After these preparations we define the appropriate notion of weak solution. We stress that our formulation uses test fields that are tangential to the target manifold of the holonomic constraint so that the multiplier $\lambda$ in (2.12) disappears; see [21] for conditions under which both formulations are equivalent.

Definition 2.1 (weak solution). Given a time-horizon $T>0$ and a bounded, convex Lipschitz domain $\Omega \subset \mathbb{R}^{2}$ we say that the pair $(q, u)$ is a weak solution of $(2.12)$ in the time interval $I=(0, T)$ subject to the initial conditions (2.13) and the boundary conditions (2.14) and (2.15) if

(i) $q \in H^{1}\left(I ; L^{2}\left(\Omega ; \mathbb{R}^{2}\right)\right) \cap L^{\infty}\left(I ; H^{1}\left(\Omega ; \mathbb{R}^{2}\right)\right), \quad u \in H^{1}\left(I ; L^{2}(\Omega)\right) \cap L^{\infty}\left(I ; H^{2}(\Omega)\right)$;

(ii) $|q(t, x)|=1$ for almost every $(t, x) \in I \times \Omega$;

(iii) $q(0, \cdot)=q_{0}, u(0, \cdot)=u_{0}$

(iv) $\left.q(t, \cdot)\right|_{\Gamma}=q_{\mathrm{D}}$ and $\left.u(t, \cdot)\right|_{\Gamma}=0$ in the sense of traces for almost every $t \in I$;

(v) for all $(\psi, \phi) \in L^{2}\left(I ; H_{0}^{1}\left(\Omega ; \mathbb{R}^{2}\right)\right) \times L^{2}\left(I ; H^{2}(\Omega) \cap H_{0}^{1}(\Omega)\right)$ satisfying $\psi \cdot q=0$ almost everywhere in $I \times \Omega$ we have

$$
\begin{aligned}
& \int_{I}\left\{\frac{1}{\gamma_{q}}\left(\partial_{t} q, \psi\right)+\mu(\nabla q, \nabla \psi)-\delta(\nabla u, \operatorname{Div} A \psi)\right\} \mathrm{d} t=0, \\
& \int_{I}\left\{\frac{1}{\gamma_{u}}\left(\partial_{t} u, \phi\right)+\kappa(\Delta u, \Delta \phi)-\delta(\operatorname{Div} A q, \nabla \phi)\right\} \mathrm{d} t=0 ;
\end{aligned}
$$

(vi) for almost every $T^{\prime} \in I$ and $E=L$ as in (2.9) with $g=0$ we have

$$
E\left[u\left(T^{\prime}, \cdot\right), q\left(T^{\prime}, \cdot\right)\right]+\frac{1}{\gamma_{q}} \int_{0}^{T^{\prime}}\left\|\partial_{t} q\right\|^{2} \mathrm{~d} t+\frac{1}{\gamma_{u}} \int_{0}^{T^{\prime}}\left\|\partial_{t} u\right\|^{2} \mathrm{~d} t \leq E\left[u_{0}, q_{0}\right]
$$

Remark 2.1 (harmonic maps). The definition of a weak solution, particularly the energy inequality (vi), is motivated by the notion of weak solutions for the harmonic map heat flow problem discussed in [11,40].

Remark 2.2 (coercivity). We assume that $\Omega$ is convex in order to guarantee that the biharmonic operator defines an elliptic bilinear form on $H^{2}(\Omega) \cap H_{0}^{1}(\Omega)$. The coercivity of the associated form follows then with the $H^{2}$ regularity of the Laplace operator subject to homogeneous Dirichlet conditions on $\Gamma$. 
Remark 2.3 (test functions). Restricting to test functions $\psi$ that are pointwise orthogonal to $q$ reflects the fact that only tangential perturbations of $q$ are permitted in the energy minimization. Including a Lagrange multiplier $\lambda$ in the formulation allows one to consider unconstrained vector fields $\psi \in L^{2}\left(I ; H_{0}^{1}\left(\Omega ; \mathbb{R}^{2}\right)\right) \cap L^{\infty}(I \times$ $\Omega ; \mathbb{R}^{2}$ ) in item (v) of Definition 2.1.

We informally summarize our results in the following theorem; see Theorem 5.2 below for the precise statement. In particular, the weak convergence is understood as the weak convergence of a suitable time-continuous interpolation on $(0, T) \times \Omega$; see Lemma 5.1 for details.

Theorem 2.2 (existence). Suppose that $\Omega \subset \mathbb{R}^{2}$ is a convex polygonal domain and that the elastic moduli and the coupling constant satisfy $\delta^{2} \leq \mu \kappa / 8 C_{P}^{2}$, where $C_{P}$ is the Poincaré constant in $H_{0}^{1}(\Omega)$. Then there exists a weak solution of the system (2.12) with initial conditions (2.13) and boundary conditions (2.14). Moreover, $\Delta u \in L^{2}\left(I ; H_{0}^{1}(\Omega)\right)$ and the boundary condition $\Delta u=0$ holds in the sense of trace.

More precisely, we show the existence of a weak solution $(u, q)$ that arises as a limit of the following fully discrete scheme for the system (2.12).

\subsection{Discretization}

The following algorithm is partially motivated by recent work in $[1-3,5]$. Here $q_{h} \in\left[\mathbb{V}_{h}\right]^{2}$ and $u_{h} \in \mathbb{V}_{h}$ are approximations to $q$ and $u$ with Courant elements, $\Delta_{h}^{0} u_{h}$ denotes a discrete analog of the Laplace operator defined on the finite element space, and $\mathcal{F}_{h}$ is the space of finite element functions that are tangential to the manifold $|q|=1$ at all nodes. We refer to Section 3 and (3.3) for the definitions and, in particular, (3.1) and (3.2) for the two types of backward difference quotients which we use.

Algorithm 2.3. Choose $\left(q_{h}^{0}, u_{h}^{0}\right) \in\left[\mathbb{V}_{h}\right]^{2} \times \mathbb{V}_{h}^{0}$ such that $\left|q_{h}^{0}(z)\right|=1$ for all $z \in \mathcal{N}_{h},\left.q_{h}^{0}\right|_{\Gamma}=q_{\mathrm{D}, h}$. Set $n=0$.

(1) Compute $\widetilde{q}_{h}^{n+1} \in q_{h}^{n}+\mathcal{F}_{h}\left[q_{h}^{n}\right]$ such that

$$
\frac{1}{\gamma_{q}}\left(\widetilde{d}_{\tau}^{-} \widetilde{q}_{h}^{n+1}, \psi_{h}\right)_{h}+\mu\left(\nabla \widetilde{q}_{h}^{n+1}, \nabla \psi_{h}\right)=\delta\left(\nabla u_{h}^{n}, \operatorname{Div} A \psi_{h}\right)
$$

for all $\psi_{h} \in \mathcal{F}_{h}\left[q_{h}^{n}\right]$.

(2) Define $q_{h}^{n+1} \in\left[\mathbb{V}_{h}\right]^{2}$ satisfying the constraint $\left|q_{h}^{n+1}\right|=1$ at the nodes by

$$
q_{h}^{n+1}(z)=\frac{\widetilde{q}_{h}^{n+1}(z)}{\left|\widetilde{q}_{h}^{n+1}(z)\right|} \quad \text { for all } z \in \mathcal{N}_{h} .
$$

(3) Compute $u_{h}^{n+1} \in \mathbb{V}_{h}^{0}$ such that

$$
\frac{1}{\gamma_{u}}\left(d_{\tau}^{-} u_{h}^{n+1}, \phi_{h}\right)_{h}+\kappa\left(\Delta_{h}^{0} u_{h}^{n+1}, \Delta_{h}^{0} \phi_{h}\right)_{h}=\delta\left(\operatorname{Div} A q_{h}^{n+1}, \nabla \phi_{h}\right)
$$

for all $\phi_{h} \in \mathbb{V}_{h}^{0}$.

(4) Stop if $n+1 \geq T / \tau$; set $n=n+1$ and go to (1) otherwise.

Remark 2.4 (nodal constraint). Note that we are committing variational crimes [13] in the sense that we impose the constraint on the length of the director and on the orthogonality of the test fields only at the nodes of the triangulation.

Remark 2.5 (stability). Discretizing explicitly the term on the right-hand side in the equation defining $\widetilde{q}_{h}^{n+1}$ does not affect the stability of the scheme. In fact, this choice relates precisely to the delay in the discrete integration (or summation) by parts formula; see (4.3) below.

The next theorem summarizes the properties of the numerical scheme. 
Theorem 2.4 (convergence). Suppose that the assumptions of Theorem 2.2 hold. Then there exists a weak solution which can be obtained as a limit of solutions of a fully discrete semi-implicit numerical scheme. More precisely, suppose that $\mathcal{T}_{h}, h>0$ is a family of regular and weakly acute triangulations of $\Omega$ and that $q_{h}^{0} \rightarrow q_{0}$ and $u_{h}^{0} \rightarrow u_{0}$ in $H^{1}\left(\Omega ; \mathbb{R}^{2}\right)$ and $H^{1}(\Omega)$, respectively. Then there exists a constant $C_{1}$ that depends only on $\Omega$ and the geometry of $\mathcal{T}_{h}$ with the following property. Suppose that $\tau^{1-\rho} \leq C_{1}^{-1} \mu \gamma_{q}^{-1} \delta^{-2} C_{0}^{-2}$ for some fixed $\rho \in(0,1)$, where $C_{0}>0$ is chosen in such a way that the initial conditions satisfy the discrete energy estimate

$$
E_{h}\left[u_{h}^{0}, q_{h}^{0}\right] \leq \frac{\kappa}{4} C_{0}^{2} \quad \text { for all } h>0 .
$$

Hereafter, the discrete energy is given by

$$
E_{h}\left[u_{h}^{n}, q_{h}^{n}\right]=\int_{\Omega} \frac{\mu}{2}\left|\nabla q_{h}^{n}\right|^{2} \mathrm{~d} x+\frac{\kappa}{2}\left(\Delta_{h}^{0} u_{h}^{n}, \Delta_{h}^{0} u_{h}^{n}\right)_{h}-\int_{\Omega} \delta \nabla u_{h}^{n} \cdot \operatorname{Div} A q_{h}^{n} \mathrm{~d} x .
$$

Then for any sequence $(h, \tau) \rightarrow 0$ of meshsizes and time-steps there exists a subsequence $(h, \tau)$ and corresponding solutions $q_{h}^{n}$ and $u_{h}^{n}$ for $n=0,1,2, \ldots, N_{T}=\lfloor T / \tau\rfloor+1$ such that $\left(q_{h}^{n}, u_{h}^{n}\right)$ converges to a weak solution $(q, u)$ as $(h, \tau) \rightarrow 0$.

Remark 2.6 (finite energy). The assumption that the initial conditions have finite energy implies a restriction on the class of admissible initial director fields $q_{h}^{0}$. We will come back to this issue in Section 6 .

Remark 2.7 (uniqueness). In general it is an open problem to prove uniqueness of solutions, even for the heat flow of harmonic maps. However, smooth solutions are unique in the class of all smooth solutions. This fact follows directly from a Gronwall argument provided that $q(t) \in W^{1, \infty}\left(\Omega ; \mathbb{R}^{2}\right)$ and $u(t) \in W^{2, \infty}(\Omega)$ uniformly for $t \in[0, T]$.

We split the analysis of Algorithm 2.3 into two parts. In Section 4 we study its stability properties, whereas in Section 5 we prove convergence to a weak solution.

\section{Finite element spaCes, operators, and Discrete estimates}

In the following we assume that $\mathcal{T}_{h}$ is a regular triangulation [13] of $\Omega$ into triangles of maximal diameter $h>0$. We denote by $\mathbb{V}_{h}$ the space of all globally continuous functions that are affine on the elements in the triangulation and we set $\mathbb{V}_{h}^{0}=\mathbb{V}_{h} \cap H_{0}^{1}(\Omega)$. We say that $\mathcal{T}_{h}$ is weakly acute if the sum of every pair of angles opposite to an interior edge is bounded by $\pi$. For a fixed time-step size $\tau>0$ we define $t_{n}=n \tau$ for all $n \geq 0$ and for a sequence of functions $\left(\phi^{n}\right)_{n \geq 0}$ we use the backward difference quotients in time,

$$
d_{\tau}^{-} \phi^{n+1}=\tau^{-1}\left(\phi^{n+1}-\phi^{n}\right) .
$$

Moreover, if $\left(\widetilde{\phi}^{n}\right)_{n \geq 0}$ is a second sequence of functions such that $\phi^{n}$ is computed from $\phi^{n-1}$ via the auxiliary function $\widetilde{\phi}^{n}$, then we set

$$
\widetilde{d}_{\tau}^{-} \widetilde{\phi}_{n}=\tau^{-1}\left(\widetilde{\phi}^{n}-\phi^{n-1}\right)
$$

Given $q_{h} \in\left[\mathbb{V}_{h}\right]^{2}$ we define the space of tangential updates at the nodes for a given vector field $q_{h}$ by

$$
\mathcal{F}_{h}\left[q_{h}\right]=\left\{r_{h} \in\left[\mathbb{V}_{h}^{0}\right]^{2}: r_{h}(z) \cdot q_{h}(z)=0 \quad \text { for all } z \in \mathcal{N}_{h}\right\}
$$


where $\mathcal{N}_{h}$ denotes the set of nodes in $\mathcal{T}_{h}$. We use the standard nodal interpolation operator $\mathcal{I}_{h}: C(\bar{\Omega}) \rightarrow \mathbb{V}_{h}$ to define a discrete inner product (also called mass lumping)

$$
(\phi, \chi)_{h}=\int_{\Omega} \mathcal{I}_{h}[\phi \cdot \chi] \mathrm{d} x \quad \text { for } \phi, \chi \in C\left(\bar{\Omega} ; \mathbb{R}^{\ell}\right), \ell \geq 1,
$$

and we set $\|\cdot\|_{h}=(\cdot, \cdot)_{h}^{1 / 2}$.

In the following we repeatedly use the well known estimate

$$
\left|\left(\psi_{h}, \phi_{h}\right)-\left(\psi_{h}, \phi_{h}\right)_{h}\right| \leq C h\left\|\psi_{h}\right\|\left\|\nabla \phi_{h}\right\|
$$

which holds for all $\phi_{h}, \psi_{h} \in \mathbb{V}_{h}$ as well as the equivalence

$$
\left\|\phi_{h}\right\| \leq\left\|\phi_{h}\right\|_{h} \leq 2\left\|\phi_{h}\right\|
$$

see, e.g., [23]. It is convenient to introduce discrete analogs of differential operators.

Definition 3.1. Let $u_{h} \in \mathbb{V}_{h}^{0}$. Then the discrete Laplace operator with homogeneous boundary conditions $\Delta_{h}^{0} u_{h} \in \mathbb{V}_{h}^{0}$ is defined through

$$
\left(\Delta_{h}^{0} u_{h}, \phi_{h}\right)_{h}=-\left(\nabla u_{h}, \nabla \phi_{h}\right) \quad \text { for all } \phi_{h} \in \mathbb{V}_{h}^{0}
$$

Moreover, we define the discrete second derivative $D_{h}^{2} u_{h} \in\left[\mathbb{V}_{h}^{0}\right]^{2 \times 2}$ of a function $u_{h} \in \mathbb{V}_{h}^{0}$ through

$$
\left(D_{h}^{2} u_{h}, P_{h}\right)_{h}=-\left(\nabla u_{h}, \operatorname{Div} P_{h}\right) \quad \text { for all } P_{h} \in\left[\mathbb{V}_{h}^{0}\right]^{2 \times 2}
$$

We also recall the set-up of a typical mixed method for the approximation of the fourth-order equation $\Delta^{2} u=f$ in its weak form,

$$
(\Delta u, \Delta \phi)=(f, \phi)
$$

for all $\phi \in H^{2}(\Omega) \cap H_{0}^{1}(\Omega)$. In this formulation, the boundary condition $\Delta u=0$ on $\Gamma$ is a natural boundary condition and a discretization is given by

$$
\begin{aligned}
\left(v_{h}, \psi_{h}\right)_{h}+\left(\nabla u_{h}, \nabla \psi_{h}\right) & =0 \\
\left(\nabla v_{h}, \nabla \phi_{h}\right) & =-\left(f, \phi_{h}\right)_{h}
\end{aligned}
$$

for all $\left(\psi_{h}, \phi_{h}\right) \in \mathbb{V}_{h}^{0} \times \mathbb{V}_{h}^{0}$. One easily verifies that the Babuška-Brezzi conditions [9] are satisfied uniformly in $h$ for this formulation. In view of Definition 3.1, the mixed method can be summarized as

$$
\left(\Delta_{h}^{0} u_{h}, \Delta_{h}^{0} \phi_{h}\right)_{h}=\left(f, \phi_{h}\right)_{h} \quad \forall \phi_{h} \in \mathbb{V}_{h}^{0}
$$

Imposing the boundary condition $\Delta_{h}^{0} u_{h}=0$ on $\Gamma$ explicitly seems necessary to guarantee stability of the discretization with lowest order elements.

An important property of the discrete Laplace operator is the following estimate

$$
\left\|\nabla v_{h}\right\| \leq 2 C_{P}\left\|\Delta_{h}^{0} v_{h}\right\|_{h} \quad \text { for all } v_{h} \in \mathbb{V}_{h}^{0}
$$

where $C_{P}>0$ is the usual Poincaré constant. In fact, for all $v_{h} \in \mathbb{V}_{h}^{0}$, we have that

$$
\left\|\nabla v_{h}\right\|^{2}=-\left(\Delta_{h}^{0} v_{h}, v_{h}\right)_{h} \leq\left\|\Delta_{h}^{0} v_{h}\right\|_{h}\left\|v_{h}\right\|_{h}=2\left\|\Delta_{h}^{0} v_{h}\right\|_{h}\left\|v_{h}\right\| \leq 2 C_{P}\left\|\Delta_{h}^{0} v_{h}\right\|_{h}\left\|\nabla v_{h}\right\| .
$$

We let $\left.q_{\mathrm{D}, h} \in \mathbb{V}_{h}^{2}\right|_{\Gamma}$ denote an approximation of $q_{\mathrm{D}}$. 
Lemma 3.2 (anti-quasi-Ritz projection). Given $v_{h} \in \mathbb{V}_{h}^{0}$ let $v \in H_{0}^{1}(\Omega)$ be the weak solution of $-\Delta v=-\Delta_{h}^{0} v_{h}$, i.e., $(\nabla v, \nabla \phi)=-\left(\Delta_{h}^{0} v_{h}, \phi\right)$ for all $\phi \in H_{0}^{1}(\Omega)$. Then we have

$$
\left\|\nabla\left[v-v_{h}\right]\right\| \leq C h\left\|D^{2} v\right\|+C h\left\|\Delta_{h}^{0} v_{h}\right\|_{h}
$$

and

$$
\left\|D^{2} v\right\| \leq C\left\|\Delta_{h}^{0} v_{h}\right\|_{h} .
$$

Proof. Let $R_{h} v \in \mathbb{V}_{h}^{0}$ be the Ritz projection of $v$, i.e.,

$$
\left(\nabla R_{h} v, \nabla \phi_{h}\right)=\left(\nabla v, \nabla \phi_{h}\right)
$$

holds for all $\phi_{h} \in \mathbb{V}_{h}^{0}$. Then, using the definition of $\Delta_{h}^{0}$, the $H^{2}$ regularity of the Laplace operator for the solution $v$, and (3.4), we have for all $\phi_{h} \in \mathbb{V}_{h}^{0}$ that

$$
\begin{aligned}
\left(\nabla\left[R_{h} v-v_{h}\right], \nabla \phi_{h}\right) & =\left(\nabla v, \nabla \phi_{h}\right)-\left(\nabla v_{h}, \nabla \phi_{h}\right)=-\left(\Delta v, \phi_{h}\right)+\left(\Delta_{h}^{0} v_{h}, \phi_{h}\right)_{h} \\
& =-\left(\Delta_{h}^{0} v_{h}, \phi_{h}\right)+\left(\Delta_{h}^{0} v_{h}, \phi_{h}\right)_{h} \leq C h\left\|\Delta_{h}^{0} v_{h}\right\|\left\|\nabla \phi_{h}\right\|
\end{aligned}
$$

and thus, upon choosing $\phi_{h}=R_{h} v-v_{h}$ and and using standard estimates for the Ritz projection [13],

$$
\left\|\nabla\left(v-v_{h}\right)\right\| \leq\left\|\nabla\left(v-R_{h} v\right)\right\|+\left\|\nabla\left(R_{h} v-v_{h}\right)\right\| \leq C h\left\|D^{2} v\right\|+C h\left\|\Delta_{h}^{0} v_{h}\right\| .
$$

The second estimate follows from the definition of $v$ and $H^{2}$ regularity of the Laplace operator in convex domains [22].

Remark 3.1 (quadrature). Note that $v_{h}$ is not the exact Ritz projection $R_{h} v$ of $v$ in Lemma 3.2 since the definition of $\Delta_{h}^{0}$ involves numerical integration.

Lemma 3.3 (discrete $H^{2}$ regularity). For all $v_{h} \in \mathbb{V}_{h}^{0}$ and $P_{h} \in\left[\mathbb{V}_{h}^{0}\right]^{2 \times 2}$ we have

$$
\left(\nabla v_{h}, \operatorname{Div} P_{h}\right) \leq C\left\|\Delta_{h}^{0} v_{h}\right\|_{h}\left\|P_{h}\right\|_{h} .
$$

In particular, we have for every $v_{h} \in \mathbb{V}_{h}^{0}$ and $D_{h}^{2} v_{h}$ defined in (3.6) that

$$
\left\|D_{h}^{2} v_{h}\right\|_{h} \leq C\left\|\Delta_{h}^{0} v_{h}\right\|_{h} .
$$

Proof. Let $v \in H_{0}^{1}(\Omega)$ be as in Lemma 3.2. Then, with the bounds of Lemma 3.2 and the inverse estimate on finite dimensional spaces [13] we infer

$$
\begin{aligned}
\left(\nabla v_{h}, \text { Div } P_{h}\right) & =\left(\nabla\left[v_{h}-v\right], \text { Div } P_{h}\right)+\left(\nabla v, \text { Div } P_{h}\right)=\left(\nabla\left[v_{h}-v\right], \text { Div } P_{h}\right)-\left(D^{2} v, P_{h}\right) \\
& \leq C h\left\|\Delta_{h}^{0} v\right\|\left\|\operatorname{Div} P_{h}\right\|+\left\|\Delta_{h}^{0} v\right\|\left\|P_{h}\right\| \leq C\left\|\Delta_{h}^{0} v\right\|\left\|P_{h}\right\|,
\end{aligned}
$$

which implies the first assertion. The second estimate follows from the first one by choosing $P_{h}=D_{h}^{2} v_{h}$.

Lemma 3.4 (monotonicity [5]). Suppose that $\mathcal{T}_{h}$ is weakly acute. Then for every $\widetilde{q}_{h} \in\left[\mathbb{V}_{h}\right]^{2}$ with $\left|\widetilde{q}_{h}(z)\right|=1$ for all $z \in \mathcal{N}_{h} \cap \Gamma$ and $\left|\widetilde{q}_{h}(z)\right| \geq 1$ for all $z \in \mathcal{N}_{h}$ the function $q_{h} \in\left[\mathbb{V}_{h}\right]^{2}$ defined by

$$
q_{h}(z)=\frac{\widetilde{q}_{h}(z)}{\left|\widetilde{q}_{h}(z)\right|}
$$

for all $z \in \mathcal{N}_{h}$ satisfies

$$
\left\|\nabla q_{h}\right\| \leq\left\|\nabla \widetilde{q}_{h}\right\|
$$


Proof. Under the assumption of the lemma the finite element stiffness matrix $\mathbb{K}=\left(k_{z y}\right)_{z, y \in \mathcal{N}_{h}}$ defined through the nodal basis $\left(\varphi_{z}: z \in \mathcal{N}_{h}\right)$ satisfies $k_{z y}=\left(\nabla \varphi_{z}, \nabla \varphi_{y}\right) \leq 0$ whenever $z \neq y$ and the line segment connecting $z$ and $y$ is not an edge on $\Gamma$. With the fact that the sum of the entries of every row of $\mathbb{K}$ vanishes and the symmetry of $\mathbb{K}$ we infer that

$$
\begin{aligned}
\left\|\nabla q_{h}\right\|^{2} & =\sum_{z, y \in \mathcal{N}_{h}} k_{z y} q_{h}(z) \cdot q_{h}(y)=\sum_{z, y \in \mathcal{N}_{h}} k_{z y} q_{h}(z) \cdot\left(q_{h}(y)-q_{h}(z)\right) \\
& =\frac{1}{2} \sum_{z, y \in \mathcal{N}_{h}} k_{z y} q_{h}(z) \cdot\left(q_{h}(y)-q_{h}(z)\right)+\frac{1}{2} \sum_{z, y \in \mathcal{N}_{h}} k_{z y} q_{h}(y) \cdot\left(q_{h}(z)-q_{h}(y)\right) \\
& =-\frac{1}{2} \sum_{z, y \in \mathcal{N}_{h}} k_{z y}\left|q_{h}(y)-q_{h}(z)\right|^{2} .
\end{aligned}
$$

Lipschitz continuity with constant 1 of the projection $q \mapsto q /|q|$ for $|q| \geq 1, q_{h}(z)=\widetilde{q}_{h}(z)$ for all $z \in \mathcal{N}_{h} \cap \Gamma$, and the properties of the entries of $\mathbb{K}$ imply

$$
-k_{z y}\left|q_{h}(y)-q_{h}(z)\right| \leq-k_{z y}\left|\widetilde{q}_{h}(y)-\widetilde{q}_{h}(z)\right|
$$

for all $z, y \in \mathcal{N}_{h}$ and this implies the assertion.

\section{UNCONDITIONAL STABILITY}

In Lemma 4.1 and Proposition 4.2 we verify unconditional well-posedness and stability of Algorithm 2.3.

Lemma 4.1 (threshold). Suppose that

$$
\delta^{2} \leq \frac{\mu \kappa}{8 C_{P}^{2}}
$$

where $C_{P}$ is the Poincaré constant in (3.7). If $E_{h}\left[u_{h}, q_{h}\right]$ is the discrete energy given by (2.16), then, for every $\left(q_{h}, u_{h}\right) \in\left[\mathbb{V}_{h}\right]^{2} \times \mathbb{V}_{h}^{0}$, we have that

$$
\frac{\kappa}{4}\left\|\Delta_{h}^{0} u_{h}\right\|_{h}^{2}+\frac{\mu}{4}\left\|\nabla q_{h}\right\|^{2} \leq E_{h}\left[u_{h}, q_{h}\right]
$$

Proof. Using (3.7) and $\left\|\operatorname{Div} A q_{h}\right\|^{2} \leq \frac{1}{2}\left\|\nabla q_{h}\right\|^{2}$ we have

$$
\left|\delta \int_{\Omega} \nabla u_{h} \cdot \operatorname{Div} A q_{h} \mathrm{~d} x\right| \leq \frac{\kappa}{16 C_{P}^{2}}\left\|\nabla u_{h}\right\|^{2}+\frac{\delta^{2} 4 C_{P}^{2}}{\kappa}\left\|\operatorname{Div} A q_{h}\right\|^{2} \leq \frac{\kappa}{4}\left\|\Delta_{h}^{0} u_{h}\right\|_{h}^{2}+\frac{2 \delta^{2} C_{P}^{2}}{\kappa}\left\|\nabla q_{h}\right\|^{2} .
$$

By (4.1) the factor in front of the second term on the right-hand side is less than or equal to $\mu / 4$. Thus the modulus of (4.2) in the energy $E_{h}\left[u_{h}, q_{h}\right]$ does not exceed half the size of the two positive terms in (2.16) and this implies the assertion.

Remark 4.1 (threshold for the square). Since the smallest eigenvalue $\lambda_{1}$ of the Laplacian is explicit for $\Omega=(0,1)^{2}$, and satisfies $\lambda_{1}=C_{P}^{-2}$, we obtain $C_{P}=\frac{1}{\sqrt{2} \pi}$.

Proposition 4.2 (weak stability). All steps of Algorithm 2.3 are well defined. Moreover, if $(4.1)$ holds, if $\mathcal{T}_{h}$ is weakly acute, and if $\tau^{1-\rho} \leq C_{1}^{-1} \mu \delta^{-2} \gamma_{q}^{-1} C_{0}^{-2}$ for some $\rho \in(0,1)$ and where the constants $C_{0}, C_{1}>0$ are chosen in such way that

$$
E_{h}\left[u_{h}^{0}, q_{h}^{0}\right] \leq \frac{\kappa}{4} C_{0}^{2}
$$


and $C_{1}$ depends only on $\Omega$ and the geometry of $\mathcal{T}_{h}$, then the inequality

$$
E_{h}\left[u_{h}^{N+1}, q_{h}^{N+1}\right]+\left(1-\tau^{\rho}\right) \tau \sum_{n=0}^{N} \frac{1}{\gamma_{q}}\left\|\widetilde{d}_{\tau}^{-} \widetilde{q}_{h}^{n+1}\right\|_{h}^{2}+\tau \sum_{n=0}^{N} \frac{1}{\gamma_{u}}\left\|d_{\tau}^{-} u_{h}^{n+1}\right\|_{h}^{2}+\tau \sum_{n=0}^{N} \frac{\mu \tau}{4}\left\|\widetilde{d}_{\tau}^{-} \nabla \widetilde{q}_{h}^{n+1}\right\|^{2} \leq E_{h}\left[u_{h}^{0}, q_{h}^{0}\right]
$$

holds for $N=0,1,2, \ldots, N_{T}-1=\lfloor T / \tau\rfloor$.

Remark 4.2 (parameter $\rho$ ). The positive parameter $\rho$ is needed to guarantee the energy inequality stated in item (vi) of Definition 2.1 as $(h, \tau) \rightarrow 0$; see Remark 2.1 and Step 3 in the proof of Theorem 5.2 below.

Proof. We divide the proof in several steps.

Step 1. The scheme is well-defined. The existence of a unique $\widetilde{q}_{h}^{n+1}$ in Step (1) of Algorithm 2.3 follows from the Lax-Milgram theorem and the fact that $\mathcal{F}_{h}\left[q_{h}^{n}\right]$ is a subspace of $\left[\mathbb{V}_{h}^{0}\right]^{2}$. Since $\left|q_{h}^{n}(z)\right|=1$ for all $z \in \mathcal{N}_{h}$ and $\widetilde{q}_{h}^{n+1}=q_{h}^{n}+r_{h}^{n}$ for some $r_{h} \in\left[\mathbb{V}_{h}^{0}\right]^{2}$ such that $q_{h}^{n}(z) \cdot r_{h}(z)=0$ for all $z \in \mathcal{N}_{h}$, we have $\left|\widetilde{q}_{h}^{n+1}(z)\right| \geq 1$ for all $z \in \mathcal{N}_{h}$ and hence the projection in Step (2) of Algorithm 2.3 is well defined. Finally, the existence of a unique $u_{h}^{n+1} \in \mathbb{V}_{h}^{0}$ in Step (3) follows from the Lax-Milgram theorem. Note that the corresponding bilinear form is uniformly coercive (independent of $h$ ) since the norm of the gradient is controlled by the discrete Laplacian; see (3.7).

Step 2. Derivation of estimates from the weak equations. In order to prove the discrete energy inequality we choose $\psi_{h}=\widetilde{d}_{\tau}^{-} \widetilde{q}_{h}^{n+1}$ and $\phi_{h}=d_{\tau}^{-} u_{h}^{n+1}$ in the equations of Step (1) and (3) in the definition of the algorithm, respectively, and obtain

$$
\frac{1}{\gamma_{q}}\left\|\widetilde{d}_{\tau}^{-} \widetilde{q}_{h}^{n+1}\right\|_{h}^{2}+\frac{\mu}{2} \widetilde{d}_{\tau}^{-}\left\|\nabla \widetilde{q}_{h}^{n+1}\right\|^{2}+\frac{\mu}{2} \tau\left\|\widetilde{d}_{\tau}^{-} \nabla \widetilde{q}_{h}^{n+1}\right\|^{2}-\delta\left(\nabla u_{h}^{n}, d_{\tau}^{-} \operatorname{Div} A q_{h}^{n+1}\right)=\delta\left(\nabla u_{h}^{n}, \operatorname{Div} A\left[\widetilde{d}_{\tau}^{-} \widetilde{q}_{h}^{n+1}-d_{\tau}^{-} q_{h}^{n+1}\right]\right)
$$

and

$$
\frac{1}{\gamma_{u}}\left\|d_{\tau}^{-} u_{h}^{n+1}\right\|_{h}^{2}+\frac{\kappa}{2} d_{\tau}^{-}\left\|\Delta_{h}^{0} u_{h}^{n+1}\right\|_{h}^{2}+\frac{\kappa \tau}{2}\left\|d_{\tau}^{-} \Delta_{h}^{0} u_{h}^{n+1}\right\|_{h}^{2}-\delta\left(d_{\tau}^{-} \nabla u_{h}^{n+1}, \operatorname{Div} A q_{h}^{n+1}\right)=0
$$

These identities will be used together with the following discrete analogue of the integration by parts formula,

$$
\tau \sum_{n=0}^{N}\left(d_{\tau}^{-} \nabla u_{h}^{n+1}, \operatorname{Div} A q_{h}^{n+1}\right)+\tau \sum_{n=0}^{N}\left(\nabla u_{h}^{n}, d_{\tau}^{-} \operatorname{Div} A q_{h}^{n+1}\right)=\left(\nabla u_{h}^{N+1}, \operatorname{Div} A q_{h}^{N+1}\right)-\left(\nabla u_{h}^{0}, \operatorname{Div} A q_{h}^{0}\right)
$$

If we multiply the foregoing identities by $\tau$ and take the sum over $n=0,1,2, \ldots, N$ then we find with (4.3) that

$$
\begin{aligned}
& \frac{\mu}{2} \max \left\{\left\|\nabla \widetilde{q}_{h}^{N+1}\right\|^{2},\left\|\nabla q_{h}^{N+1}\right\|^{2}\right\}+\frac{\kappa}{2}\left\|\Delta_{h}^{0} u_{h}^{N+1}\right\|_{h}^{2}-\delta\left(\nabla u_{h}^{N+1}, \operatorname{Div} A q_{h}^{N+1}\right) \\
& +\tau \sum_{n=0}^{N} \frac{1}{\gamma_{q}}\left\|\widetilde{d}_{\tau}^{-} \widetilde{q}_{h}^{n+1}\right\|_{h}^{2}+\tau \sum_{n=0}^{N} \frac{1}{\gamma_{u}}\left\|d_{\tau}^{-} u_{h}^{n+1}\right\|_{h}^{2}+\tau \sum_{n=0}^{N} \frac{\mu \tau}{2}\left\|\widetilde{d}_{\tau}^{-} \nabla \widetilde{q}_{h}^{n+1}\right\|^{2}+\tau \sum_{n=0}^{N} \frac{\kappa \tau}{2}\left\|d_{\tau}^{-} \Delta_{h}^{0} u_{h}^{n+1}\right\|_{h}^{2} \\
& \leq \frac{\mu}{2}\left\|\nabla q_{h}^{0}\right\|^{2}+\frac{\kappa}{2}\left\|\Delta_{h}^{0} u_{h}^{0}\right\|_{h}^{2}-\delta\left(\nabla u_{h}^{0}, \operatorname{Div} A q_{h}^{0}\right)+\tau \sum_{n=0}^{N} \delta\left(\nabla u_{h}^{n}, \operatorname{Div} A\left[\widetilde{d}_{\tau}^{-} \widetilde{q}_{h}^{n+1}-d_{\tau}^{-} q_{h}^{n+1}\right]\right) .
\end{aligned}
$$

Here we used the estimate $\left\|\nabla q_{h}^{n+1}\right\| \leq\left\|\nabla \widetilde{q}_{h}^{n+1}\right\|$ guaranteed by Lemma 3.4 in order to estimate $\left\|\nabla q_{h}^{N+1}\right\|$. However, we will use the stronger estimate with $\nabla q_{h}^{N+1}$ in (5.3) below. 
Step 3. Estimates for the coupling term. To estimate the last term on the right-hand side we notice that $A\left[\widetilde{d}_{\tau}^{-} \widetilde{q}_{h}^{n+1}-d_{\tau}^{-} q_{h}^{n+1}\right] \in\left[\mathbb{V}_{h}^{0}\right]^{2 \times 2}$ and employ Lemma 3.3 to deduce that

$$
\left(\nabla u_{h}^{n}, \operatorname{Div} A\left[\widetilde{d}_{\tau}^{-} \widetilde{q}_{h}^{n+1}-d_{\tau}^{-} q_{h}^{n+1}\right]\right) \leq C\left\|\Delta_{h}^{0} u_{h}^{n}\right\|_{h}\left\|A\left[\widetilde{d}_{\tau}^{-} \widetilde{q}_{h}^{n+1}-d_{\tau}^{-} q_{h}^{n+1}\right]\right\|_{h}
$$

We directly verify for every $z \in \mathcal{N}_{h}$ the identity

$$
\left|A\left[\widetilde{d}_{\tau}^{-} \widetilde{q}_{h}^{n+1}-d_{\tau}^{-} q_{h}^{n+1}\right](z)\right|=\tau^{-1}\left|A \widetilde{q}_{h}^{n+1}(z)-\frac{A \widetilde{q}_{h}^{n+1}(z)}{\left|\widetilde{q}_{h}^{n+1}(z)\right|}\right|=\tau^{-1} \frac{\left|A \widetilde{q}_{h}^{n+1}(z)\right|}{\left|\widetilde{q}_{h}^{n+1}(z)\right|}\left(\left|\widetilde{q}_{h}^{n+1}(z)\right|-1\right) .
$$

Since $\widetilde{q}_{h}^{n+1}(z)=q_{h}^{n}(z)+\tau \widetilde{d}_{\tau}^{-} \widetilde{q}_{h}^{n+1}(z), q_{h}^{n}(z) \cdot \widetilde{d}_{\tau}^{-} \widetilde{q}_{h}^{n+1}(z)=0$, and $\left|q_{h}^{n}(z)\right|=1$ for all $z \in \mathcal{N}_{h}$ we obtain that

$$
\left|\widetilde{q}_{h}^{n+1}(z)\right|-1=\left(\left|q_{h}^{n}(z)\right|^{2}+\tau^{2}\left|\widetilde{d}_{\tau}^{-} \widetilde{q}_{h}^{n+1}(z)\right|^{2}\right)^{1 / 2}-1=\left(1+\tau^{2}\left|\widetilde{d}_{\tau}^{-} \widetilde{q}_{h}^{n+1}(z)\right|^{2}\right)^{1 / 2}-1 \leq \frac{1}{2} \tau^{2}\left|\widetilde{d}_{\tau}^{-} \widetilde{q}_{h}^{n+1}(z)\right|^{2}
$$

where we used $\left(1+t^{2}\right)^{1 / 2} \leq 1+t^{2} / 2$ for all $t \in \mathbb{R}$ in the last estimate. For future reference we note that the foregoing estimates imply

$$
\left|A\left[\widetilde{d}_{\tau}^{-} \widetilde{q}_{h}^{n+1}-d_{\tau}^{-} q_{h}^{n+1}\right](z)\right| \leq C \tau\left|\widetilde{d}_{\tau}^{-} \widetilde{q}_{h}^{n+1}(z)\right|^{2} \quad \text { for all } z \in \mathcal{N}_{h} .
$$

With the definition of $\|\cdot\|_{h}$ and appropriate coefficients $\left(\beta_{z}^{h}: z \in \mathcal{N}_{h}\right)$ such that $\left\|v_{h}\right\|_{h}^{2}=\sum_{z \in \mathcal{N}_{h}} \beta_{z}^{h}\left|v_{h}(z)\right|^{2}$ for every $v_{h} \in \mathbb{V}_{h}$ we infer

$$
\begin{aligned}
\left\|A\left[\widetilde{d}_{\tau}^{-} \widetilde{q}_{h}^{n+1}-d_{\tau}^{-} q_{h}^{n+1}\right]\right\|_{h}^{2} & =\sum_{z \in \mathcal{N}_{h}} \beta_{z}^{h}\left|A\left[\widetilde{d}_{\tau}^{-} \widetilde{q}_{h}^{n+1}-d_{\tau}^{-} q_{h}^{n+1}\right](z)\right|^{2} \\
& \leq \frac{1}{4} \tau^{2}\|A\|^{2} \sum_{z \in \mathcal{N}_{h}} \beta_{z}^{h}\left|\widetilde{d}_{\tau}^{-} \widetilde{q}_{h}^{n+1}(z)\right|^{4} \leq C \tau^{2}\left\|\widetilde{d}_{\tau}^{-} \widetilde{q}_{h}^{n+1}\right\|_{L^{4}(\Omega)}^{4}
\end{aligned}
$$

where we used that $\sum_{z \in \mathcal{N}_{h}} \beta_{z}^{h}\left|v_{h}(z)\right|^{4} \leq C\left\|v_{h}\right\|_{L^{4}(\Omega)}^{4}$. In view of the interpolation estimate [28], p. 45, there exists a constant $C=C(\Omega)$ such that

$$
\|v\|_{L^{4}(\Omega)}^{2} \leq C\|v\|\|\nabla v\| \quad \text { for } v \in H_{0}^{1}(\Omega) .
$$

We next apply Young's inequality, together with (3.5), to deduce that

$$
\begin{aligned}
\delta\left(\nabla u_{h}^{n}, \operatorname{Div} A\left[\widetilde{d}_{\tau}^{-} \widetilde{q}_{h}^{n+1}-d_{\tau}^{-} q_{h}^{n+1}\right]\right) \leq C \tau \delta & \left\|\Delta_{h}^{0} u_{h}^{n}\right\|_{h}\left\|\widetilde{d}_{\tau}^{-} \widetilde{q}_{h}^{n+1}\right\|\left\|\widetilde{d}_{\tau}^{-} \nabla \widetilde{q}_{h}^{n+1}\right\| \\
& \leq \frac{\tau^{\rho}}{\gamma_{q}}\left\|\widetilde{d}_{\tau}^{-} \widetilde{q}_{h}^{n+1}\right\|_{h}^{2}+\left(C_{1} \gamma_{q} \mu^{-1} \delta^{2} \tau^{1-\rho}\left\|\Delta_{h}^{0} u_{h}^{n}\right\|_{h}^{2}\right) \frac{\mu}{4} \tau\left\|\widetilde{d}_{\tau}^{-} \nabla \widetilde{q}_{h}^{n+1}\right\|^{2} .
\end{aligned}
$$

Step 4. Verification of the discrete energy inequality. We are now going to verify by induction that

$$
1 \max _{n=0,1, \ldots, N}\left\|\Delta_{h}^{0} u_{h}^{n}\right\|_{h} \leq C_{0} \quad \text { for all } N=0,1, \ldots, N_{T}
$$

and that the discrete energy inequality follows under this assumption for $N+1$ from the corresponding statement for $N$.

The estimate (4.7) is clearly true for $N=0$ since $u_{h}^{0}=0$. Suppose next that this estimate is true for some $N \geq 0$. Since by assumption

$$
\tau^{1-\rho} \leq C_{1}^{-1} \mu \delta^{-2} \gamma_{q}^{-1} C_{0}^{2}
$$


we obtain

$$
C_{1} \gamma_{q} \mu^{-1} \delta^{2} \tau^{1-\rho}\left\|\Delta_{h}^{0} u_{h}^{n}\right\|_{h}^{2} \leq 1
$$

for $n=0,1, \ldots, N$ and infer, upon combining (4.6) and (4.4), that

$$
\begin{array}{r}
1 \frac{\mu}{2}\left\|\nabla q_{h}^{N+1}\right\|^{2}+\frac{\kappa}{2}\left\|\Delta_{h}^{0} u_{h}^{N+1}\right\|_{h}^{2}-\delta\left(\nabla u_{h}^{N+1}, \operatorname{Div} A q_{h}^{N+1}\right)+\left(1-\tau^{\rho}\right) \tau \sum_{n=0}^{N} \frac{1}{\gamma_{q}}\left\|\widetilde{d}_{\tau}^{-} \widetilde{q}_{h}^{n+1}\right\|_{h}^{2}+\tau \sum_{n=0}^{N} \frac{1}{\gamma_{u}}\left\|d_{\tau}^{-} u_{h}^{n+1}\right\|_{h}^{2} \\
+\tau \sum_{n=0}^{N} \frac{\mu \tau}{4}\left\|\widetilde{d}_{\tau}^{-} \nabla \widetilde{q}_{h}^{n+1}\right\|^{2} \leq \frac{\mu}{2}\left\|\nabla q_{h}^{0}\right\|^{2}+\frac{\kappa}{2}\left\|\Delta_{h}^{0} u_{h}^{0}\right\|_{h}^{2}-\delta\left(\nabla u_{h}^{0}, \operatorname{Div} A q_{h}^{0}\right)=E_{h}\left[u_{h}^{0}, q_{h}^{0}\right] .
\end{array}
$$

This completes the inductive proof for the discrete energy estimate. It remains to prove that (4.7) is true for $N+1$. However, since the left-hand side is an upper bound for $E_{h}\left[u_{h}^{N+1}, q_{h}^{N+1}\right]$ we conclude

$$
\frac{\kappa}{4}\left\|\Delta_{h}^{0} u_{h}^{N+1}\right\|_{h}^{2} \leq E_{h}\left[u_{h}^{N+1}, q_{h}^{N+1}\right] \leq E_{h}\left[u_{h}^{0}, q_{h}^{0}\right] \leq \frac{\kappa}{4} C_{0}^{2}
$$

and therefore $\left\|\Delta_{h}^{0} u_{h}^{N+1}\right\| \leq C_{0}$. This establishes the induction step and the assertion for all $N$.

The following stronger estimate will be important in the compactness proof.

Proposition 4.3 (strong stability). Suppose that the assumptions of Proposition 4.2 are satisfied. Then we have for every $N=0,1,2, \ldots, N_{T}-1$ that

$$
\frac{1}{2 \gamma_{u}}\left\|\nabla u_{h}^{N+1}\right\|^{2}+\tau \sum_{n=0}^{N} \frac{\tau}{2 \gamma_{u}}\left\|d_{\tau}^{-} \nabla u_{h}^{n+1}\right\|^{2}+\tau \sum_{n=0}^{N} \frac{\kappa}{2}\left\|\nabla \Delta_{h}^{0} u_{h}^{n+1}\right\|^{2} \leq C N \tau .
$$

Proof. We choose $\phi_{h}=-\Delta_{h}^{0} u_{h}^{n+1} \in \mathbb{V}_{h}^{0}$ in step (3) of Algorithm 2.3. This leads to

$$
\frac{1}{2 \gamma_{u}} d_{\tau}^{-}\left\|\nabla u_{h}^{n+1}\right\|^{2}+\frac{\tau}{2 \gamma_{u}}\left\|d_{\tau}^{-} \nabla u_{h}^{n+1}\right\|^{2}+\kappa\left\|\nabla \Delta_{h}^{0} u_{h}^{n+1}\right\|^{2}=\delta\left(\operatorname{Div} A q_{h}^{n+1}, \nabla \Delta_{h}^{0} u_{h}^{n+1}\right) .
$$

We use Young's inequality to bound the right-hand side as

$$
\delta\left(\operatorname{Div} A q_{h}^{n+1}, \nabla \Delta_{h}^{0} u_{h}^{n+1}\right) \leq \frac{\delta^{2}}{2 \kappa}\left\|\operatorname{Div} A q_{h}^{n+1}\right\|^{2}+\frac{\kappa}{2}\left\|\nabla \Delta_{h}^{0} u_{h}^{n+1}\right\|^{2}
$$

Proposition 4.2 implies $\left\|\operatorname{Div} A q_{h}^{n+1}\right\| \leq C_{A}\left\|\nabla q_{h}^{n+1}\right\| \leq C$ and the assertion follows after multiplication of (4.8) by $\tau$, summing the resulting inequalities from $n=0,1,2, \ldots, N$, and recalling that $u_{h}^{0}=0$.

\section{Convergence}

Our convergence analysis is based on compactness theorems in Sobolev spaces together with a priori bounds that follow from the discrete estimates. For $t_{n}<t \leq t_{n+1}$ we define piecewise constant interpolants of the iterates of Algorithm 2.3 through

$$
\begin{array}{rlrl}
\widetilde{q}_{h}(t) & =\widetilde{d}_{\tau}^{-} \widetilde{q}_{h}^{n+1}, & \widetilde{q}_{h}(t) & =\widetilde{q}_{h}^{n+1}, \\
u_{h}^{R}(t) & =u_{h}^{n+1}, & q_{h}(t) & =q_{h}^{n+1}, \\
q_{h}^{n}, & u_{h}^{R}(t) & =u_{h}^{n},
\end{array}
$$

and piecewise affine approximations via

$$
\widehat{u}_{h}(t)=u_{h}^{n}+\left(t-t_{n}\right) d_{\tau}^{-} u_{h}^{n+1}, \quad \widehat{q}_{h}(t)=q_{h}^{n}+\left(t-t_{n}\right) d_{\tau}^{-} q_{h}^{n+1} .
$$


In terms of these functions we may rewrite the equations of Algorithm 2.3 as

$$
\begin{array}{r}
\frac{1}{\gamma_{q}}\left(\widetilde{q}_{h}, \psi_{h}\right)_{h}+\mu\left(\nabla \widetilde{q}_{h}, \nabla \psi_{h}\right)-\delta\left(\nabla u_{h}^{R}, \operatorname{Div} A \psi_{h}\right)=0, \\
\frac{1}{\gamma_{u}}\left(\partial_{t} \widehat{u}_{h}, \phi_{h}\right)_{h}+\kappa\left(\Delta_{h}^{0} u_{h}, \Delta_{h}^{0} \phi_{h}\right)_{h}-\delta\left(\operatorname{Div} A q_{h}, \nabla \phi_{h}\right)=0
\end{array}
$$

for almost every $t \in I$, for all $\psi_{h} \in \mathcal{F}_{h}\left[q_{h}^{R}(t, \cdot)\right]$, and for all $\phi_{h} \in \mathbb{V}_{h}^{0}$.

The bounds in Propositions 4.2 and 4.3 allow us to select weakly convergent subsequences with the following properties.

Lemma 5.1 (weak convergence). Suppose that the assumptions of Proposition 4.2 are satisfied and that $q_{h}^{0} \rightarrow q_{0}$ in $H^{1}\left(\Omega ; \mathbb{R}^{2}\right)$. Then there exists a subsequence $(h, \tau) \rightarrow 0$ and a pair $(q, u)$ with

$$
\begin{aligned}
\widetilde{q}_{h}, \partial_{t} \widehat{q}_{h} \rightarrow \partial_{t} q & \text { in } L^{2}\left(I ; L^{2}\left(\Omega ; \mathbb{R}^{2}\right)\right), \\
\widehat{q}_{h}, \widetilde{q}_{h}, q_{h}, q_{h}^{R} \stackrel{*}{\rightarrow} q & \text { in } L^{\infty}\left(I ; H^{1}\left(\Omega ; \mathbb{R}^{2}\right)\right), \\
\partial_{t} \widehat{u}_{h} \rightarrow \partial_{t} u & \text { in } L^{2}\left(I ; L^{2}(\Omega)\right), \\
u_{h}, u_{h}^{R} \stackrel{*}{\rightarrow} u & \text { in } L^{\infty}\left(I ; H_{0}^{1}(\Omega)\right), \\
\Delta_{h}^{0} u_{h} \rightarrow \Delta u & \text { in } L^{2}\left(I ; H_{0}^{1}(\Omega)\right), \\
D_{h}^{2} u_{h}, D_{h}^{2} u_{h}^{R} \stackrel{*}{\rightarrow} D^{2} u & \text { in } L^{\infty}\left(I ; L^{2}\left(\Omega ; \mathbb{R}^{2 \times 2}\right)\right) .
\end{aligned}
$$

Moreover, $|q|=1$ almost everywhere in $I \times \Omega, q(0, \cdot)=q_{0}$ as well as $u(0, \cdot)=0$, and $\left.q(t, \cdot)\right|_{\Gamma}=q_{\mathrm{D}}$ in the sense of traces for almost every $t \in I$.

Proof. We first establish the convergence properties for $q_{h}$, then verify that the limit $q$ satisfies the constraint, and finally prove the convergence for $u_{h}$. All of these results hold for suitably chosen subsequences.

Step 1. Convergence of $q_{h}$. From the bounds in Proposition 4.2, the estimate (4.4), the constraint on the length of $q_{h}$ in the nodes, and Poincaré's inequality we immediately deduce that

$$
q_{h}, \widetilde{q}_{h} \in L^{\infty}\left(I ; H^{1}\left(\Omega ; \mathbb{R}^{2}\right)\right), \quad \widetilde{q}_{h} \in L^{2}\left(I ; L^{2}\left(\Omega ; \mathbb{R}^{2}\right)\right)
$$

and that the sequences are uniformly bounded in these space. Thus there exists a $q \in L^{\infty}\left(I ; H^{1}\left(\Omega ; \mathbb{R}^{2}\right)\right)$ and a $q^{\prime} \in L^{2}\left(I ; L^{2}\left(\Omega ; \mathbb{R}^{2}\right)\right)$ such that $q_{h} \stackrel{*}{\rightarrow} q$ in $L^{\infty}\left(I ; H^{1}\left(\Omega ; \mathbb{R}^{2}\right)\right)$ and $\widetilde{q}_{h}^{\prime} \rightarrow q^{\prime}$ in $L^{2}\left(I ; L^{2}\left(\Omega ; \mathbb{R}^{2}\right)\right)$, respectively. We verify in (5.5) below that $q^{\prime}$ is indeed the time derivative of $q$. In order to verify the second assertion in $(a)$ we note that the estimate (4.5) implies

$$
\left\|\widetilde{q}_{h}-\partial_{t} \widehat{q}_{h}\right\|_{L^{1}(I \times \Omega)} \leq C \tau\left\|\widetilde{q}_{h}^{\prime}\right\|_{L^{2}(I \times \Omega)}^{2}
$$

and the right-hand side of this inequality tends to zero as $(h, \tau) \rightarrow 0$ since the $L^{2}$ norms are uniformly bounded. It remains to prove a uniform $L^{2}$ bound. It follows from $\left(1+t^{2}\right)^{1 / 2} \leq 1+t^{2} / 2$ together with $\left|q_{h}^{n}(z)\right|=1$ and $q_{h}^{n}(z) \cdot \widetilde{d}_{\tau}^{-} \widetilde{q}_{h}^{n+1}(z)=0$ that for all $z \in \mathcal{N}_{h}$

$$
\begin{aligned}
\left|q_{h}^{n+1}(z)-q_{h}^{n}(z)\right|^{2} & =\left|\frac{q_{h}^{n}(z)+\tau \widetilde{d}_{\tau}^{-} \widetilde{q}_{h}^{n+1}(z)}{\left|q_{h}^{n}(z)+\tau \widetilde{d}_{\tau}^{-} \widetilde{q}_{h}^{n+1}(z)\right|}-q_{h}^{n}(z)\right|^{2} \\
& =2-\frac{2}{\left|q_{h}^{n}(z)+\tau \widetilde{d}_{\tau}^{-} \widetilde{q}_{h}^{n+1}(z)\right|} \leq 2\left(\left|q_{h}^{n}(z)+\tau \widetilde{d}_{\tau}^{-} \widetilde{q}_{h}^{n+1}(z)\right|-1\right) \\
& =2\left(\left(1+\tau^{2}\left|\widetilde{q}_{h}^{n+1}(z)\right|^{2}\right)^{1 / 2}-1\right) \leq \tau^{2}\left|\widetilde{q}_{h}^{n+1}(z)\right|^{2}
\end{aligned}
$$


and this implies that $\partial_{t} \widehat{q}_{h}$ is uniformly bounded in $L^{2}\left(I \times \Omega ; \mathbb{R}^{2}\right)$. Hence there exists a weakly convergent subsequence, and since weak limits are unique we deduce from $(5.4)$ that $\partial_{t} \widehat{q}_{h} \rightarrow q^{\prime}$ in $L^{2}\left(I ; L^{2}\left(\Omega ; \mathbb{R}^{2}\right)\right)$. We finally observe that for every $\phi \in C_{0}^{\infty}(I \times \Omega)$ we have

$$
\int_{I}\left(q, \partial_{t} \phi\right) \mathrm{d} t=\lim _{(h, \tau) \rightarrow 0} \int_{I}\left(\widehat{q}_{h}, \partial_{t} \phi\right) \mathrm{d} t=-\lim _{(h, \tau) \rightarrow 0} \int_{I}\left(\partial_{t} \widehat{q}_{h}, \phi\right)=-\int_{I}\left(q^{\prime}, \phi\right) \mathrm{d} t,
$$

i.e., $\partial_{t} q=q^{\prime}$.

To prove the assertions in $(b)$, we first observe that the convergence of the piecewise constant functions $q_{h}$ to $q$ and the boundedness of $\partial_{t} \widehat{q}_{h}$ in $L^{2}\left(I ; L^{2}\left(\Omega ; \mathbb{R}^{2}\right)\right)$ imply $\widehat{q}_{h}, q_{h}^{R} \stackrel{*}{\rightarrow} q$ in $L^{\infty}\left(I ; H^{1}(\Omega)\right)$ as well. Thus we only need to show the convergence of $\widetilde{q}_{h}$. Arguing as above we find that

$$
\left\|\widetilde{q}_{h}-q_{h}\right\|_{L^{1}(I \times \Omega)} \leq C \tau^{2}\left\|\widetilde{q}_{h}^{\prime}\right\|_{L^{2}(I \times \Omega)}^{2}
$$

so that also $\widetilde{q}_{h} \stackrel{*}{\rightarrow} q$ in $L^{\infty}\left(I ; H^{1}\left(\Omega ; \mathbb{R}^{2}\right)\right)$.

Step 2. Validity of the constraint. We first observe that

$$
\widehat{q}_{h} \in L^{2}\left(I ; H^{1}\left(\Omega ; \mathbb{R}^{2}\right)\right), \quad \partial_{t} \widehat{q}_{h} \in L^{2}\left(I ; L^{2}\left(\Omega ; \mathbb{R}^{2}\right)\right) \subset L^{2}\left(I ; H^{-1}\left(\Omega ; \mathbb{R}^{2}\right)\right)
$$

with uniform bounds in these space by $(b)$ and $(a)$, respectively. Hence the Aubin-Lions lemma implies that

$$
\widehat{q}_{h} \rightarrow q \quad \text { in } L^{2}(I \times \Omega) .
$$

Since we have additionally $\widehat{q}_{h}-q_{h} \rightarrow 0$ in $L^{2}(I \times \Omega)$ we have pointwise convergence $q_{h} \rightarrow q$ almost everywhere in $I \times \Omega$. The fact that $\left|q_{h}(t, z)\right|=1$ for all $z \in \mathcal{N}_{h}$ and almost every $t \in I$ implies the following estimate. Let $K$ be an element of the triangulation $x \in K$ and let $z$ be one of the nodes in $K$. Then

$$
\begin{aligned}
\left|q_{h}(t, x)\right|^{2}-1 & =\left|q_{h}(t, x)\right|^{2}-\left|q_{h}(t, z)\right|^{2}=\int_{0}^{1} \frac{\mathrm{d}}{\mathrm{d} s}\left|q_{h}(t, s x+(1-s) z)\right|^{2} \mathrm{~d} s \\
& =2 \int_{0}^{1}\left(\nabla q_{h}(t, s x+(1-s) z)\right):\left(q_{h}(t, s x+(1-s) z) \otimes(x-z)\right) \mathrm{d} s
\end{aligned}
$$

and hence

$$
\left.|| q_{h}(t, x)\right|^{2}-1|\leq c h| \nabla q_{h}(t, x) \mid \quad \text { for all } x \in K
$$

If we integrate the inequality in space-time, then

$$
\int_{0}^{T}\left\|\left.|| q_{h}(t, \cdot)\right|^{2}-1\right\|^{2} \mathrm{~d} t \leq C h^{2} \int_{0}^{T}\left\|\nabla q_{h}(t, \cdot)\right\|^{2} \mathrm{~d} t
$$

and we obtain in view of $(b)$ that $\left|q_{h}\right|^{2} \rightarrow 1$ in $L^{2}(I \times \Omega)$ and thus $\left|q_{h}(t, x)\right|^{2} \rightarrow 1$ for almost every $(t, x) \in I \times \Omega$. This implies $|q|=1$ almost everywhere in $I \times \Omega$. The assertion that $\left.q(t, \cdot)\right|_{\Gamma}=q_{\mathrm{D}}$ for almost every $t \in I$ follows from weak continuity properties of the trace operator. Finally, $q(0, \cdot)=q_{0}$ is derived with an integration by parts in time and the limits identified above.

Step 3. Convergence of $u_{h}$. It remains to prove the assertions for $u$. The bounds of Propositions 4.2 and 4.3 imply that

$$
\partial_{t} \widehat{u}_{h} \in L^{2}\left(I ; L^{2}(\Omega)\right), \quad \Delta_{h}^{0} u_{h} \in L^{\infty}\left(I ; L^{2}(\Omega)\right), \quad \nabla \Delta_{h}^{0} u_{h} \in L^{2}\left(I ; L^{2}(\Omega)\right) .
$$


In view of the Poincaré-type inequality (3.7) for the discrete Laplacian and the Poincaré inequality for $u_{h}$ and $\Delta_{h}^{0} u_{h}$ we obtain that

$$
u_{h} \in L^{\infty}\left(I ; H_{0}^{1}(\Omega)\right), \quad \Delta_{h}^{0} u_{h} \in L^{2}\left(I ; H_{0}^{1}(\Omega)\right) .
$$

Since the sequences are uniformly bounded in these spaces we infer the existence of a function $u \in H^{1}\left(I ; L^{2}(\Omega)\right) \cap$ $L^{\infty}\left(I ; H_{0}^{1}(\Omega)\right)$ with

$$
u_{h} \stackrel{*}{\rightarrow} u \text { in } L^{\infty}\left(I ; H_{0}^{1}(\Omega)\right), \quad \partial_{t} \widehat{u}_{h} \rightarrow \partial_{t} u \text { in } L^{2}(I \times \Omega)
$$

and a function $\chi \in L^{2}\left(I ; H_{0}^{1}(\Omega)\right)$ with

$$
\Delta_{h}^{0} u_{h} \rightarrow \chi \text { in } L^{2}\left(I ; H_{0}^{1}(\Omega)\right)
$$

respectively. The boundedness of $\partial_{t} \widehat{u}_{h}$ implies $u_{h}-u_{h}^{R} \rightarrow 0$ in $L^{2}(I \times \Omega)$ and hence $u_{h}^{R} \stackrel{*}{\rightarrow} u$ in $L^{\infty}\left(I ; H_{0}^{1}(\Omega)\right)$. This verifies $(c)$ and $(d)$. We show next that $(e)$, i.e., $\chi=\Delta u$. With the definition of $\Delta_{h}^{0} u_{h}$ and (3.4) we have for every $\phi \in C_{0}^{\infty}(I \times \Omega)$ that

$$
\begin{aligned}
\int_{I}(\chi, \phi) \mathrm{d} t & =\lim _{(h, \tau) \rightarrow 0} \int_{I}\left(\Delta_{h}^{0} u_{h}, \phi\right) \mathrm{d} t=\lim _{(h, \tau) \rightarrow 0} \int_{I}\left(\Delta_{h}^{0} u_{h}, \phi\right)_{h} \mathrm{~d} t \\
& =-\lim _{(h, \tau) \rightarrow 0} \int_{I}\left(\nabla u_{h}, \nabla \mathcal{I}_{h} \phi\right) \mathrm{d} t=-\int_{I}(\nabla u, \nabla \phi) \mathrm{d} t
\end{aligned}
$$

and this proves $\chi=\Delta u$. Finally, to prove $(f)$ note that the boundedness of $\Delta_{h}^{0} u_{h}$ in $L^{\infty}\left(I ; L^{2}(\Omega)\right)$ implies also that $\Delta_{h}^{0} u_{h}^{R}$ is bounded in $L^{\infty}\left(I ; L^{2}(\Omega)\right)$ and with Lemma 3.3 we find that

$$
D_{h}^{2} u_{h}, D_{h}^{2} u_{h}^{R} \in L^{\infty}\left(I ; L^{2}\left(\Omega ; \mathbb{R}^{2 \times 2}\right)\right)
$$

together with a uniform bound in this space. Hence, there exists a $P, P^{R} \in L^{\infty}\left(I ; L^{2}\left(\Omega ; \mathbb{R}^{2 \times 2}\right)\right)$ such that (up to a subsequence) $D_{h}^{2} u_{h}^{R} \stackrel{*}{\rightarrow} P$ and $D_{h}^{2} u_{h}^{R} \stackrel{*}{\rightarrow} P^{R}$, respectively. We want to show that $P=P^{R}=D^{2} u$. For every $\psi \in C_{0}^{\infty}\left(I \times \Omega ; \mathbb{R}^{2 \times 2}\right)$ we deduce in view of the definition of $D_{h}^{2} u_{h}$, the convergence $(d)$ and with $\left\|\nabla\left[\mathcal{I}_{h} \psi-\psi\right]\right\|_{L^{2}(I \times \Omega)} \rightarrow 0$, that

$$
\int_{0}^{T}(P, \psi) \mathrm{d} t=\lim _{(h, \tau) \rightarrow 0} \int_{I}\left(D_{h}^{2} u_{h}, \psi\right)_{h} \mathrm{~d} t=-\lim _{(h, \tau) \rightarrow 0} \int_{I}\left(\nabla u_{h}, \operatorname{Div} \mathcal{I}_{h} \psi\right) \mathrm{d} t=-\int_{I}(\nabla u, \operatorname{Div} \psi) \mathrm{d} t
$$

which proves $P=D^{2} u$. The fact that $u(0, \cdot)=0$ follows again from integrating by parts in time. The assertion $P^{R}=D^{2} u$ follows with the same argument since $u_{h}^{R} \stackrel{*}{\rightarrow} u$ in $L^{\infty}\left(I ; H_{0}^{1}(\Omega)\right)$, and hence

$$
\int_{0}^{T}(P, \psi) \mathrm{d} t=-\lim _{(h, \tau) \rightarrow 0} \int_{I}\left(\nabla u_{h}^{R}, \operatorname{Div} \mathcal{I}_{h} \psi\right) \mathrm{d} t=-\int_{I}(\nabla u, \operatorname{Div} \psi) \mathrm{d} t
$$

This concludes the proof.

We are now in position to pass to the limit in equations (5.1) and (5.2).

Theorem 5.2 (existence). Under the assumptions of Lemma 5.1 the pair $(q, u)$ from Lemma 5.1 is a weak solution of (2.12) in the sense of Definition 2.1. 
Proof. We first deal with the equation for the deformation $u$, then with the equation for the director field, in which we need to pay attention to the constraint $|q|=1$. The last step is then the verification of the energy inequality for the solution $(u, q)$.

Step 1. Passage to the limit in the equation for $u$. Let $\phi \in C_{0}^{\infty}(I \times \Omega)$ and set $\phi_{h}(t, \cdot)=\mathcal{I}_{h} \phi(t, \cdot)$ for all $t \in I$. We want to pass to the limit $(h, \tau) \rightarrow 0$ in the integrated version of equation (5.2). For the first term we write

$$
\int_{I}\left(\partial_{t} \widehat{u}_{h}, \phi_{h}\right)_{h} \mathrm{~d} t=\int_{I}\left(\partial_{t} \widehat{u}_{h}, \phi_{h}\right) \mathrm{d} t+\int_{I}\left\{\left(\partial_{t} \widehat{u}_{h}, \phi_{h}\right)_{h}-\left(\partial_{t} \widehat{u}_{h}, \phi_{h}\right)\right\} \mathrm{d} t .
$$

Since by Lemma 5.1(c) $\partial_{t} \widehat{u}_{h} \rightarrow \partial_{t} u$ in $L^{2}(I \times \Omega)$, and since by general interpolation estimates $\phi_{h} \rightarrow \phi$ in $L^{2}(I \times \Omega)$, we obtain together with $(3.4)$ and $\left\|\nabla \phi_{h}\right\|_{L^{2}(I \times \Omega)} \leq C_{\phi}$ that, as $(h, \tau) \rightarrow 0$,

$$
\int_{I}\left(\partial_{t} \widehat{u}_{h}, \phi_{h}\right)_{h} \mathrm{~d} t \rightarrow \int_{I}\left(\partial_{t} u, \phi\right) \mathrm{d} t
$$

Here we write $C_{\phi}$ for a constant that depends on $\phi$ but is independent on $h$ and all the other parameters. The definition of $\Delta_{h}^{0}$ and the facts that $\Delta_{h}^{0} u_{h} \rightarrow \Delta u$ in $L^{2}\left(I ; H_{0}^{1}(\Omega)\right)$ and $\nabla \phi_{h} \rightarrow \nabla \phi$ in $L^{2}(I \times \Omega)$ imply that

$$
\int_{I}\left(\Delta_{h}^{0} u_{h}, \Delta_{h}^{0} \phi_{h}\right)_{h} \mathrm{~d} t=-\int_{I}\left(\nabla \Delta_{h}^{0} u_{h}, \nabla \phi_{h}\right) \mathrm{d} t \rightarrow-\int_{I}(\nabla \Delta u, \nabla \phi) \mathrm{d} t=\int_{I}(\Delta u, \Delta \phi) \mathrm{d} t
$$

as $(h, \tau) \rightarrow 0$. Note that by Lemma $5.1 \Delta u=0$ on $\partial \Omega$. Since $q_{h} \rightarrow q$ in $L^{2}\left(I ; H^{1}\left(\Omega ; \mathbb{R}^{2}\right)\right)$ we verify that

$$
\int_{I}\left(\operatorname{Div} A q_{h}, \nabla \phi_{h}\right) \mathrm{d} t \rightarrow \int_{I}(\operatorname{Div} A q, \nabla \phi) \mathrm{d} t
$$

as $(h, \tau) \rightarrow 0$. Combining (5.7)-(5.9) we conclude

$$
\int_{I}\left\{\frac{1}{\gamma_{u}}\left(\partial_{t} u, \phi\right)+\kappa(\Delta u, \Delta \phi)-\delta(\operatorname{Div} A q, \nabla \phi)\right\} \mathrm{d} t=0
$$

holds for all $\phi \in C_{0}^{\infty}(I \times \Omega)$. A density argument allows us to verify the same identity for all $\phi \in L^{2}\left(I ; H^{2}(\Omega) \cap\right.$ $\left.H_{0}^{1}(\Omega)\right)$.

Step 2. Passage to the limit in the equation for $q$. Passage to the limit in (5.1) is slightly more involved due to the constraint on the discrete test functions. Let $\xi \in C_{0}^{\infty}(I \times \Omega)$ and define

$$
\psi_{h}(t, \cdot)=\mathcal{I}_{h}\left[\xi(t, \cdot) J q_{h}^{R}(t, \cdot)\right]
$$

for every $t \in I$, where

$$
J=\left[\begin{array}{rr}
0 & -1 \\
1 & 0
\end{array}\right]
$$

denotes the counter-clockwise rotation by $\pi / 2$. With the usual convention to interpret the second gradients as acting on the functions restricted to the elements, we infer

$$
\left\|\psi_{h}-\xi J q_{h}^{R}\right\|_{L^{2}(I \times \Omega)} \leq C h^{2}\left\|\nabla^{2}\left(\xi J q_{h}^{R}\right)\right\|_{L^{2}(I \times \Omega)} \leq C h^{2}\|\xi\|_{W^{2, \infty}(I \times \Omega)}\left(\left\|q_{h}^{R}\right\|_{L^{2}(I \times \Omega)}+\left\|\nabla q_{h}^{R}\right\|_{L^{2}(I \times \Omega)}\right)
$$

and hence

$$
\lim _{(h, \tau) \rightarrow 0}\left\|\psi_{h}-\xi J q_{h}^{R}\right\|_{L^{2}(I \times \Omega)}=0 .
$$


Moreover $\psi_{h}(t, z) \cdot q_{h}^{R}(t, z)=0$ for almost every $t \in I$ and every $z \in \mathcal{N}_{h}$ and $\psi_{h}$ is an admissible test function in (5.1). Observe next that in view of the strong convergence (5.6) and the bound (a) in Lemma 5.1

$$
\lim _{(h, \tau) \rightarrow 0}\left\|q_{h}^{R}-q\right\|_{L^{2}(I \times \Omega)}=0 .
$$

We set $\psi=\xi J q$ and conclude in view of the two foregoing estimates that

$$
\begin{aligned}
\left\|\psi_{h}-\psi\right\|_{L^{2}(I \times \Omega)} & \leq\left\|\psi_{h}-\xi J q_{h}^{R}\right\|_{L^{2}(I \times \Omega)}+\left\|\xi\left[q_{h}^{R}-q\right]\right\|_{L^{2}(I \times \Omega)} \\
& \leq h C_{\xi}\left(1+\left\|\nabla q_{h}^{R}\right\|_{L^{2}(I \times \Omega)}\right)+\|\xi\|_{L^{\infty}(I \times \Omega)}\left\|q_{h}^{R}-q\right\|_{L^{2}(I \times \Omega)} \rightarrow 0
\end{aligned}
$$

as $(h, \tau) \rightarrow 0$. Since $\left\|\nabla \psi_{h}\right\|_{L^{2}(I \times \Omega)} \leq C_{\psi}$ we obtain as before in view of the weak convergence $(a)$ in Lemma 5.1

$$
\int_{I}\left(\widetilde{q}_{h}^{\prime}, \psi_{h}\right)_{h} \mathrm{~d} t \rightarrow \int_{I}\left(\partial_{t} q, \psi\right) \mathrm{d} t
$$

as $(h, \tau) \rightarrow 0$. Using $\widetilde{q}_{h}-q_{h}^{R}=\tau \widetilde{q}_{h}$ almost everywhere in $I \times \Omega$ we have

$$
\int_{I}\left(\nabla \widetilde{q}_{h}, \nabla \psi_{h}\right)=\tau \int_{I}\left[\left(\nabla \widetilde{q}_{h}^{\prime}, \nabla \psi_{h}\right)+\left(\nabla q_{h}^{R}, \nabla\left[\psi_{h}-\xi J q_{h}^{R}\right]\right)+\left(\nabla q_{h}^{R}, \nabla\left[\xi J q_{h}^{R}\right]\right)\right] \mathrm{d} t=: T_{1}+T_{2}+T_{3} .
$$

The bound of Proposition 4.2 implies $\tau^{1 / 2}\left\|\nabla \widetilde{q}_{h}^{\prime}\right\|_{L^{2}(I \times \Omega)} \leq C$ so that

$$
\left|T_{1}\right|=\left|\tau \int_{I}\left(\nabla \widetilde{q}_{h}, \nabla \psi_{h}\right) \mathrm{d} t\right| \leq \tau\left\|\nabla \widetilde{q}_{h}\right\|_{L^{2}(I \times \Omega)}\left\|\nabla \psi_{h}\right\|_{L^{2}(I \times \Omega)} \rightarrow 0
$$

as $(h, \tau) \rightarrow 0$. The error estimates for the nodal interpolation and $\left|q_{h}^{R}\right| \leq 1$ almost everywhere imply that for almost every $t \in I$ and every $K \in \mathcal{T}_{h}$

$$
\left\|\nabla\left[\psi_{h}-\xi J q_{h}^{R}\right]\right\|_{L^{2}(K)} \leq C h\left\|\nabla^{2}\left[\xi J q_{h}^{R}\right]\right\|_{L^{2}(K)} \leq C h\left(\left\|\nabla^{2} \xi\right\|_{L^{2}(K)}+\|\nabla \xi\|_{L^{\infty}(K)}\left\|\nabla q_{h}^{R}\right\|_{L^{2}(K)}\right)
$$

and therefore we have that

$$
\left|T_{2}\right| \rightarrow 0
$$

as $(h, \tau) \rightarrow 0$. Since $J$ is a rotation by $\pi / 2$ we deduce

$$
T_{3}=\int_{I}\left(\nabla q_{h}^{R}, \nabla \xi \otimes J q_{h}^{R}+\xi \nabla\left(J q_{h}^{R}\right)\right) \mathrm{d} t=\int_{I}\left(\nabla q_{h}^{R}, \nabla \xi \otimes J q_{h}^{R}\right) \mathrm{d} t .
$$

Since $\partial_{i} q_{h}^{R} \rightarrow \partial_{i} q$ in $L^{2}\left(I \times \Omega ; \mathbb{R}^{2}\right)$ and $q_{h}^{R} \rightarrow q \in L^{2}\left(I \times \Omega ; \mathbb{R}^{2}\right)$ we may pass to the limit in this equation and reverse the argument to obtain in the limit $(h, \tau) \rightarrow 0$

$$
T_{3} \rightarrow \int_{I}(\nabla q, \nabla \xi \otimes J q) \mathrm{d} t=\int_{I}(\nabla q, \nabla[\xi J q]) \mathrm{d} t=\int_{I}(\nabla q, \nabla \psi) \mathrm{d} t .
$$

These identifications of the limits of the terms $T_{i}, i=1,2,3$ allows us to conclude that

$$
\int_{I}\left(\nabla \widetilde{q}_{h}, \nabla \psi_{h}\right) \mathrm{d} t \rightarrow \int_{I}(\nabla q, \nabla \psi) \mathrm{d} t
$$

as $(h, \tau) \rightarrow 0$. We next observe the convergence $(f)$ in Lemma 5.1 implies

$$
D_{h}^{2} u_{h}^{R} \rightarrow D^{2} u \quad \text { in } L^{2}\left(I \times \Omega ; \mathbb{R}^{2 \times 2}\right) .
$$


This fact, estimate (3.4), the definition of $D_{h}^{2} u_{h}^{R}$ and the strong convergence $A \psi_{h} \rightarrow A \psi$ in $L^{2}\left(I \times \Omega ; \mathbb{R}^{2 \times 2}\right)$ imply for $(h, \tau) \rightarrow 0$

$$
\int_{I}\left(\nabla u_{h}^{R}, \operatorname{Div} A \psi_{h}\right) \mathrm{d} t=-\int_{I}\left(D_{h}^{2} u_{h}^{R}, A \psi_{h}\right)_{h} \mathrm{~d} t \rightarrow-\int_{I}\left(D^{2} u, A \psi\right) \mathrm{d} t=\int_{I}(\nabla u, \operatorname{Div} A \psi) \mathrm{d} t .
$$

The combination of (5.10)-(5.12) proves that the identity

$$
\int_{I}\left\{\frac{1}{\gamma_{q}}\left(\partial_{t} q, \psi\right)+\mu(\nabla q, \nabla \psi)-\delta(\nabla u, \operatorname{Div} A \psi)\right\} \mathrm{d} t=0
$$

is satisfied for every $\psi$ of the form $\psi=\xi J q$ with $\xi \in C_{0}^{\infty}(I \times \Omega)$. It remains to prove this identity for all test functions $\psi \in L^{2}\left(I ; H_{0}^{1}(\Omega)\right)$. In order to do this we first observe that functions $\xi$ of the form $\xi(t, x)=\alpha(x) \beta(t)$ with $\alpha \in C_{0}^{\infty}(\Omega)$ and $\beta \in C_{0}^{\infty}(I)$ allow us to conclude that

$$
\frac{1}{\gamma_{q}}\left(\partial_{t} q, \psi\right)+\mu(\nabla q, \nabla \psi)-\delta(\nabla u, \operatorname{Div} A \psi)=0
$$

for all $\psi$ of the form $\psi(x, t)=\alpha(x) J q(t, x)$ with $\alpha \in C_{0}^{\infty}(\Omega)$ and for a.e. $t \in I$. Let $\rho \in H_{0}^{1}\left(\Omega ; \mathbb{R}^{2}\right) \cap L^{\infty}\left(\Omega ; \mathbb{R}^{2}\right)$ with $\rho \cdot q=0$ for a.e. $x \in \Omega$. Then define $\alpha \in L^{1}(\Omega)$ by $\rho(x)=\alpha(x) J q(x)$, i.e.,

$$
\alpha(x)=\rho(x) \cdot J q(x) \quad \text { a.e. } x \in \Omega \text {. }
$$

Since $|q|=1$ and $\rho \in L^{\infty}\left(\Omega ; \mathbb{R}^{2}\right)$ we have $\alpha \in L^{\infty}(\Omega)$ and the product rule for weak derivatives implies that

$$
\nabla \alpha=(\nabla \rho)^{T} J q+\rho^{T} \nabla J q \in L^{2}\left(\Omega ; \mathbb{R}^{2}\right)
$$

Since $\rho \in H_{0}^{1}(\Omega)$ we obtain $\alpha \in H_{0}^{1}(\Omega) \cap L^{\infty}(\Omega)$ and hence there exists a sequence of functions $\alpha_{\epsilon} \in C_{0}^{\infty}(\Omega)$ with $\alpha_{\epsilon} \rightarrow \alpha \in H_{0}^{1}(\Omega)$ and $\alpha_{\epsilon} \in L^{\infty}(\Omega)$ uniformly in $\epsilon$. Let $\psi_{\epsilon}=\alpha_{\epsilon} J q$ and use $\psi_{\epsilon}$ as test function in (5.13). Since

$$
\nabla \psi_{\epsilon}=\nabla \alpha_{\epsilon} J q+\alpha_{\epsilon} \nabla J q \rightarrow \nabla \alpha J q+\alpha \nabla J q=\nabla \rho
$$

we conclude that (5.13) holds for all $\psi \in H_{0}^{1}\left(\Omega ; \mathbb{R}^{2}\right) \cap L^{\infty}\left(\Omega ; \mathbb{R}^{2}\right)$ with $\psi \cdot q=0$ a.e.

We next use the techniques in [20] to remove the constraint that $\psi$ be bounded. Let $a:[0, \infty) \rightarrow[0, \infty)$ be a bounded and smooth function with $a(s)=s$ on $[0,1]$. For $R>0$ define

$$
\omega_{R}(z)=R a\left(\frac{|z|}{R}\right) \frac{z}{|z|}
$$

For any $\psi \in H_{0}^{1}(\Omega)$ with $\psi \cdot q=0$ a.e. we define $\psi_{k}=\omega_{k} \circ \psi, k \in \mathbb{N}$. Then $\psi_{k} \cdot q=0$ a.e. and $\psi_{k} \rightarrow \psi$ pointwise a.e. The chain rule implies pointwise convergence of $\left[\left(\nabla \omega_{k}\right) \circ \psi\right] \nabla \psi$ to $\nabla \psi$ together with a uniform $L^{2}$ bound and thus the same convergence in $H^{1}(\Omega)$. This allows us to use $\psi_{k}$ as a test function in (5.13) and passing to the limit we obtain this identity for all $\psi \in H_{0}^{1}(\Omega)$ with $\psi \cdot q=0$ a.e. Finally the property (v) in the definition of the weak solution in Definition (2.1) follows by approximation of functions in $L^{2}\left(I ; H_{0}^{1}(\Omega)\right)$ with simple functions of the from $\alpha(x) \beta(t)$ with finite-valued functions $\beta$.

Step 3. Verification of the energy inequality. It remains to show that item (vi) in Definition 2.1 is satisfied by the pair $(q, u)$. Since $q_{h}^{0} \rightarrow q_{0}$ in $H^{1}\left(\Omega ; \mathbb{R}^{2}\right)$ and $u_{h}^{0}=0$ we find that $E_{h}\left[u_{h}^{0}, q_{h}^{0}\right] \rightarrow E\left[u_{0}, q_{0}\right]$. By weak lower 
semi-continuity of norms and the weak convergence properties of the discrete approximations the energy law for $(q, u)$ follows from Proposition 4.2 if we can show that for almost every $t \in I$ we have

$$
\int_{\Omega} \nabla u_{h} \cdot \operatorname{Div} A q_{h} \mathrm{~d} x \rightarrow \int_{\Omega} \nabla u \cdot \operatorname{Div} A q \mathrm{~d} x
$$

as $(h, \tau) \rightarrow 0$. To verify this convergence we recall from Lemma $5.1(f)$ that $D_{h}^{2} u_{h} \stackrel{*}{\rightarrow} D^{2} u$ in $L^{\infty}\left(I ; L^{2}\left(\Omega ; \mathbb{R}^{2 \times 2}\right)\right)$ and that by $(5.6) q_{h}(t, \cdot) \rightarrow q(t, \cdot)$ in $L^{2}\left(\Omega ; \mathbb{R}^{2}\right)$ as $(h, \tau) \rightarrow 0$. Since the boundary conditions are time independent we conclude from the definition of the discrete second gradients that

$$
\begin{aligned}
\lim _{(h, \tau) \rightarrow 0} \int_{\Omega} \nabla u_{h} \cdot \operatorname{Div} A q_{h} \mathrm{~d} x & =\lim _{(h, \tau) \rightarrow 0} \int_{\Omega} \nabla u_{h} \cdot \operatorname{Div} A\left[q_{h}-q_{h}^{0}\right] \mathrm{d} x+\lim _{(h, \tau) \rightarrow 0} \int_{\Omega} \nabla u_{h} \cdot \operatorname{Div} A q_{h}^{0} \mathrm{~d} x \\
& =-\lim _{(h, \tau) \rightarrow 0}\left(D_{h}^{2} u_{h}, A\left[q_{h}-q_{h}^{0}\right]\right)_{h}+\lim _{(h, \tau) \rightarrow 0} \int_{\Omega} \nabla u_{h} \cdot \operatorname{Div} A q_{h}^{0} \mathrm{~d} x \\
& =-\left(D^{2} u, A\left[q-q_{0}\right]\right)+\int_{\Omega} \nabla u \cdot \operatorname{Div} A q_{0} \mathrm{~d} x=\int_{\Omega} \nabla u \cdot \operatorname{Div} A q \mathrm{~d} x
\end{aligned}
$$

This finishes the proof of the theorem.

\section{NumericAl EXPERIMENTS}

In this section we investigate the performance of Algorithm 2.3 in several typical test scenarios. In our simulations the domain is the unit square in the plane, $\Omega=(0,1)^{2}$, and the implementation of Algorithm 2.3 was realized in MATLAB. All systems of linear equations were solved using the backslash operator in MATLAB. The underlying triangulations $\mathcal{T}_{\ell}$ were obtained by $\ell$ uniform refinements of the coarse triangulation $\mathcal{T}^{c}=\left\{K_{1}, K_{2}\right\}$ with

$$
K_{1}=\operatorname{conv}\{(0,0),(1,0),(1,1)\}, \quad K_{2}=\operatorname{conv}\{(0,0),(1,1),(0,1)\}
$$

and $\widetilde{\mathcal{T}}_{\ell}$ by subsequent perturbation of the interior nodes of $\mathcal{T}_{\ell}$ by a random vector field of length at most $h_{\ell} / 4$, so as to avoid superconvergence effects due to high symmetry of $\mathcal{T}_{\ell}$; note that $h_{\ell}=\sqrt{2} 2^{-\ell}$ is the diameter of elements in $\mathcal{T}_{\ell}$.

This work has been partially motivated by experimental observations in [27] and by simulations reported in [41]; our simulations explore these connections. The results in [41] are based on gradient flow dynamics analogous to (2.12) for the energy $F$ in (1.2) using the parameters in (2.3) as well as

$$
T=6400, \quad \gamma_{u}^{*}=\gamma_{q}^{*}=0.1
$$

If we complement our rescaling in Section 2.1 with a nondimensionalization in time via $t=T \tau$, then the final time is 1 and the relaxation parameters are given by

$$
\gamma_{u}=\gamma_{q}=T \varepsilon^{2} \gamma_{u}^{*} \approx 2.4 \times 10^{-3}
$$

Comparing (2.2) with (2.4) and (4.1), we see that the physical coupling parameter $\delta$ must satisfy

$$
\delta^{2} \leq \varepsilon \frac{\mu \kappa}{8 C_{P}^{2}}
$$

in order to satisfy the assumptions of Lemma 4.1, and so of Theorems 2.2 and 2.4. In view of Remark 4.1, which shows that $C_{P}=1 /(\sqrt{2} \pi)$, the choice of $\delta=1$ in [41] seems to lead to a set of material parameters that is not covered by our stability and convergence analysis, which in turn require $\delta \leq 0.4391$. 


\subsection{Convergence analysis and experimental error}

Our first set of experiments concerns the numerical validation of the convergence result in Theorem 2.4. In order to experimentally study the rate of convergence of approximations obtained with our algorithm in case of smooth solutions, we introduce source terms in the evolution equations (2.12), i.e., we consider the system

$$
\begin{aligned}
& \partial_{t} u+\gamma_{u}\left(\kappa \Delta^{2} u+\delta \operatorname{div} \operatorname{Div} A q\right)=f, \\
& \partial_{t} q+\gamma_{q}\left(-\mu \Delta q+\delta A^{*} D^{2} u+\lambda q\right)=g .
\end{aligned}
$$

This allows us to choose a deformation $u$ and a corresponding order parameter $q$ and to obtain these functions as solutions of the evolution problem with the corresponding forcing $f$ and $g$. For simplicity, we identify $\mathbb{C}$ and $\mathbb{R}^{2}$ below, and choose the set of parameters

$$
T=1, \quad \gamma_{u}=\gamma_{q}=0.1, \quad \kappa=\mu=\delta=1 .
$$

For $(t, x) \in(0,1) \times(0,1)^{2}$ we define the functions

$$
\begin{aligned}
& u(t, x)=\sin (2 \pi t) \sin \left(2 \pi x_{1}\right) \sin \left(2 \pi x_{2}\right), \\
& q(t, x)=\exp \left(\mathrm{i} \cos (2 \pi t) 4 x_{1}\left(1-x_{1}\right) x_{2}\left(1-x_{2}\right)\right)
\end{aligned}
$$

and the corresponding forcing terms

$$
\begin{aligned}
& f=\partial_{t} u+\Delta^{2} u+\operatorname{div} \operatorname{Div} A q, \\
& g=\partial_{t} q-\Delta q+\left[\partial_{1}^{2} u-\partial_{2}^{2} u, 2 \partial_{12} u\right]^{T}+\lambda q,
\end{aligned}
$$

with $\lambda=-\mu|\nabla q|^{2}-\delta D^{2} u: A q$. The initial data and the boundary conditions in the algorithm are obtained by standard interpolation as $q_{h}^{0}=\mathcal{I}_{h} q(0, \cdot)$ and $q_{h, D}=\left.q_{h}^{0}\right|_{\Gamma}$, respectively. The algorithm itself was modified in order to take care of the (discrete) forcing terms which are implemented using numerical integration as in Section 3.

Table 1 summarizes our numerical results over (slightly distorted) triangulations $\widetilde{\mathcal{T}}_{\ell}$ and time-steps $\tau_{\ell}=h_{\ell} /(10 \sqrt{2})$. For each $\ell$ we display the $L^{\infty}\left(L^{2}\right)$ error of the gradient of the director field $q$, the deformation $u$, the (linearized) curvature $\Delta u$ and the energy defined by

$$
E_{h}^{n}=E_{h}\left[u_{h}^{n}, q_{h}^{n}\right], \quad \tilde{E}_{h}^{n}=E_{h}\left[\mathcal{I}_{h} u\left(t_{n}, \cdot\right), \mathcal{I}_{h} q\left(t_{n}, \cdot\right)\right]
$$

We observed that the convergence is linear in the gradient of the director and quadratic in the deformation $u$, the curvature $\Delta u$, and the total energy; see Table 1. Our theory does not provide a rate of convergence but merely guarantees weak convergence to the unique solution of our test example; see Remark 2.7.

\subsection{Ordering of random initial director field}

Our second numerical example aims at simulating the process of cooling the sample from its isotropic phase to the gel phase. This process leads to the formation of local order and enforces a uniform director field if the boundary conditions lead to an anchoring of the molecules in one fixed direction.

In order to simulate this process after quenching, we choose again the set of parameters

$$
T=1, \quad \gamma_{u}=\gamma_{q}=0.1, \quad \kappa=\mu=\delta=1
$$


TABLE 1. Errors in four significant quantities for the example in Section 6.1. Algorithm 2.3 exhibits an excellent performance beyond theory. The experimental rates of convergence (for two consecutive computations on refinement levels $\ell$ and $\ell+1$ ) are given by: 1.82, 1.5, 1.18 for the gradient of the director field; $2.5,1.97,1.95$ for the deformation; 2.33, 1.89, 1.96 for the curvature; and $2.34,1.80,1.97$ for the energy. The computations were done on meshes $\widetilde{\mathcal{T}}_{\ell}$, obtained by a random perturbation of order $\mathcal{O}\left(h_{\ell} / 4\right)$ of the meshes $\mathcal{T}_{\ell}$, in order to rule out superconvergence effects.

\begin{tabular}{|l|r|r|r|c|}
\hline \multicolumn{1}{|c|}{ Error quantity } & $\ell=3$ & $\ell=4$ & $\ell=5$ & $\ell=6$ \\
\hline \hline $\max _{n=0,1, \ldots, N}\left\|\nabla\left(q_{h}^{n}-\mathcal{I}_{h} q\left(t_{n}, \cdot\right)\right)\right\|$ & 0.4295 & 0.1218 & 0.0409 & 0.0180 \\
\hline $\max _{n=0,1, \ldots, N}\left\|u_{h}^{n}-\mathcal{I}_{h} u\left(t_{n}, \cdot\right)\right\|$ & 0.0827 & 0.0138 & 0.0035 & 0.0009 \\
\hline $\max _{n=0,1, \ldots, N}\left\|\Delta_{h}^{0} u_{h}^{n}-\mathcal{I}_{h} \Delta u\left(t_{n}, \cdot\right)\right\|$ & 2.7849 & 0.5541 & 0.1494 & 0.0384 \\
\hline $\max _{n=0,1, \ldots, N}\left|E_{h}^{n}-\tilde{E}_{h}^{n}\right|$ & 92.4940 & 18.1884 & 5.2344 & 1.3301 \\
\hline
\end{tabular}

Let $\mathcal{T}_{5}$ be the triangulation of $\Omega=(0,1)^{2}$ described above and define $\tau=h_{5} /(10 \sqrt{2})$. The initial data $q_{h}^{0} \in \mathbb{V}_{h}^{2}$ for the vector field $q$ are given by $q_{h}^{0}(z)=\left(2 n_{1}(z)^{2}-1,2 n_{1}(z) n_{2}(z)\right)$ for $z \in \mathcal{N}_{h}$ where

$$
\left(n_{1}(z), n_{2}(z)\right)= \begin{cases}\xi(z) & \text { for } z \in \Omega \\ (1,0) & \text { for } z \in \partial \Omega\end{cases}
$$

and $\xi(\cdot) \in S^{1}$ is a random vector field. We set $q_{h, D}=\left.q_{h}^{0}\right|_{\Gamma}$ and we assume that the initial configuration is a flat membrane with $u_{0} \equiv 0$. We first observe a very rapid decay of the energy at the beginning of the evolution; see Figure 1. Figure 2 shows snapshots of the displacement $u_{h}$ and the discrete director field $n_{h}$ during the evolution. For the purpose of visualization we do not plot the order parameter $q$ but the direction of alignment which can be obtained from $q$ through the formula

$$
\left(n_{1}, n_{2}\right)= \begin{cases}\frac{1}{\sqrt{2}}\left(q_{1}+1\right)^{-1 / 2}\left(q_{1}+1, q_{2}\right) & \text { if } q_{1} \neq-1, \\ (0,1) & \text { otherwise }\end{cases}
$$

(we show the direction of the director without an orientation since the energy depends only on $n \otimes n$ ). As expected, the random initial director field induces small perturbations of the initially flat membrane. However, at later stages of the evolution the director field $n$ decays to a constant field typical of the minimizing state.

\subsection{Nonflat initial states and spontaneous curvature}

In our third set of numerical experiments we include a non-vanishing initial displacement of the membrane given by the scalar function $u_{0}$ and consider compatible nonhomogeneous boundary conditions $\left.u\right|_{\Gamma}=u_{D}=\left.u_{0}\right|_{\Gamma}$ and $\left.\Delta u\right|_{\Gamma}=g=\left.\Delta u_{0}\right|_{\Gamma}$. Moreover, we extend our model to capture spontaneous curvature of the membrane [25]. That is, we do not assume that the flat membrane has zero bending energy but that due to corrugations of the substrate the ground state is no longer flat but has locally a mean curvature prescribed by a scalar function $g_{s}$, the spontaneous curvature, which depends on the position.

It is straight-forward to modify Algorithm 2.3 and to adopt the techniques presented in this paper in order to handle the additional terms in the energy. For simplicity we assume that $\left.g_{s}\right|_{\Gamma}=0$. If we write the displacement as a perturbation of the initial state, $u=\tilde{u}+u_{0}$, then the generalized energy functional is given by

$$
\widehat{E}[\tilde{u}, q]=\frac{\kappa}{2} \int_{\Omega}\left|\Delta\left(\tilde{u}+u_{0}\right)-g_{s}\right|^{2} \mathrm{~d} x+\frac{\mu}{2} \int_{\Omega}|\nabla q|^{2} \mathrm{~d} x-\kappa \int_{\Gamma} \Delta u_{0} \partial_{\nu}\left(\tilde{u}+u_{0}\right) \mathrm{d} s-\delta \int_{\Omega} \nabla\left(\tilde{u}+u_{0}\right) \cdot \operatorname{Div} A q \mathrm{~d} x .
$$




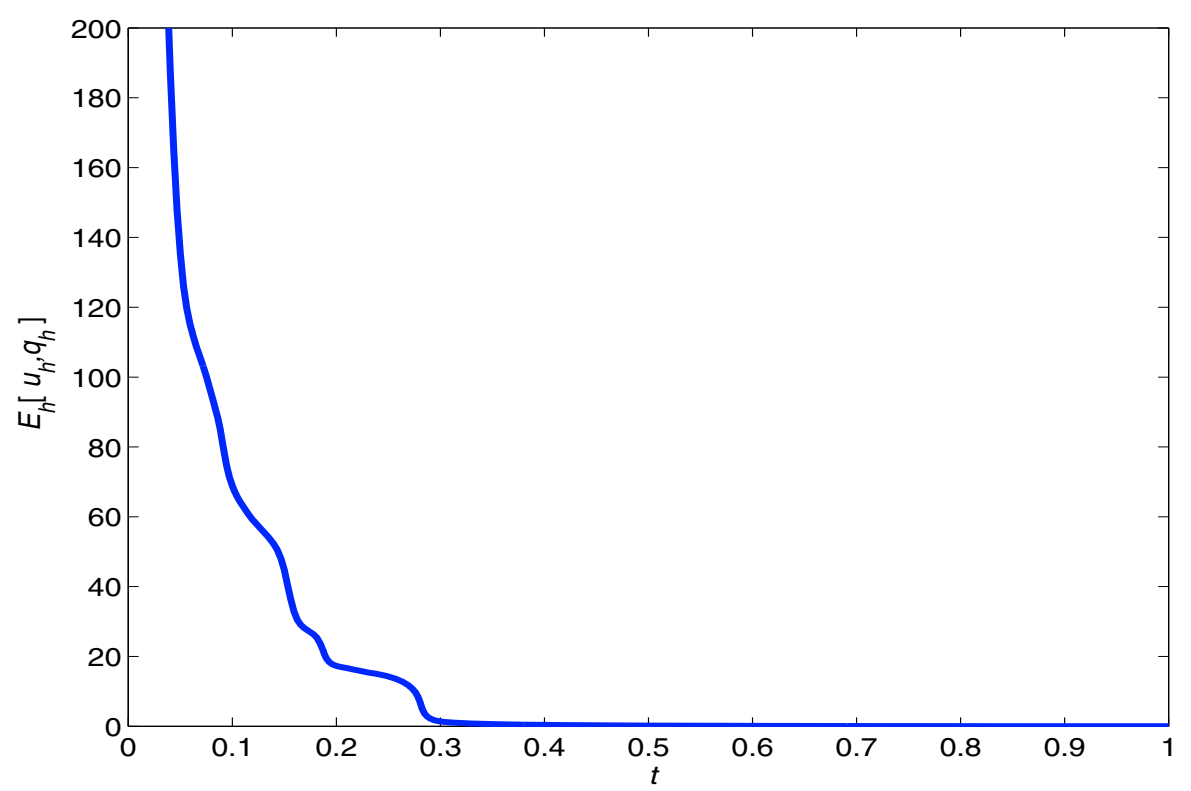

Figure 1. Rapid decay of the discrete energy $E_{h}\left[u_{h}(t), q_{h}(t)\right]$ as a function of $t \in[0,1]$ for the setting defined in Setting 6.2 with random initial data for $q$.

Integration by parts and binomial formulas show that we have

$$
\begin{aligned}
\widehat{E}[\tilde{u}, q]= & \frac{\kappa}{2} \int_{\Omega}|\Delta \tilde{u}|^{2} \mathrm{~d} x-\frac{\kappa}{2} \int_{\Omega}\left|\Delta u_{0}\right|^{2} \mathrm{~d} x+\kappa \int_{\Omega} \nabla g_{s} \cdot \nabla\left(\tilde{u}+u_{0}\right) \mathrm{d} x+\frac{\mu}{2} \int_{\Omega}|\nabla q|^{2} \mathrm{~d} x \\
& -\kappa \int_{\Omega} \nabla \Delta u_{0} \cdot \nabla\left(\tilde{u}+u_{0}\right) \mathrm{d} x-\delta \int_{\Omega} \nabla\left(\tilde{u}+u_{0}\right) \cdot \operatorname{Div} A q \mathrm{~d} x+\frac{\kappa}{2} \int_{\Omega} g_{s}^{2} \mathrm{~d} x .
\end{aligned}
$$

We set $\mu=1 / 90, \delta=1$, and $\kappa=10, \gamma_{u}=\gamma_{q}=1$, and $T=1$. We let $\mathcal{T}_{5}$ be the triangulation of $\Omega=(0,1)^{2}$ described above and define $\tau=h_{5} /(10 \sqrt{2})$. We choose $q_{h}^{0}(z) \equiv(-1,0)$ and $u_{0, h}=\mathcal{I}_{h} u_{0}, g_{s, h}=\mathcal{I}_{h} g_{s}$, where

$$
u_{0}(x)=\frac{1}{10} \sin \left(4 \pi x_{1}\right) \sin \left(4 \pi x_{2}\right), \quad g_{s}(x)=\frac{1}{2} \Delta u_{0}(x)
$$

for $x=\left(x_{1}, x_{2}\right) \in \Omega$.

Figure 3 shows the deformation of the membrane and the director field for $t=0,0.2,0.4$. As opposed to the discrete version of the energy $E$ it is in general not true that a discrete version of $\widehat{E}$, which uses interpolated functions and numerical quadrature, is non-negative. Indeed, for data defined above, the energy is negative in this example and this effect seems to be due to a rough approximation of the linear terms in the energy functional. To illustrate this, we computed for $\ell=3,4,5,6$ the quantity

$$
\xi_{\ell}=-\frac{\kappa}{2}\left\|\mathcal{I}_{h} \Delta u_{0}\right\|_{h}^{2}-\kappa \int_{\Omega} \nabla \mathcal{I}_{h} \Delta u_{0} \cdot \nabla \mathcal{I}_{h} u_{0}
$$

and obtained the values

$$
\xi_{3}=774.4, \quad \xi_{4}=1121.3, \quad \xi_{5}=1215.0, \quad \xi_{6}=1238.8 .
$$

In Figure 4 we compare the energies for different mesh-sizes and observe that they quadratically converge to a value close to 0 . 

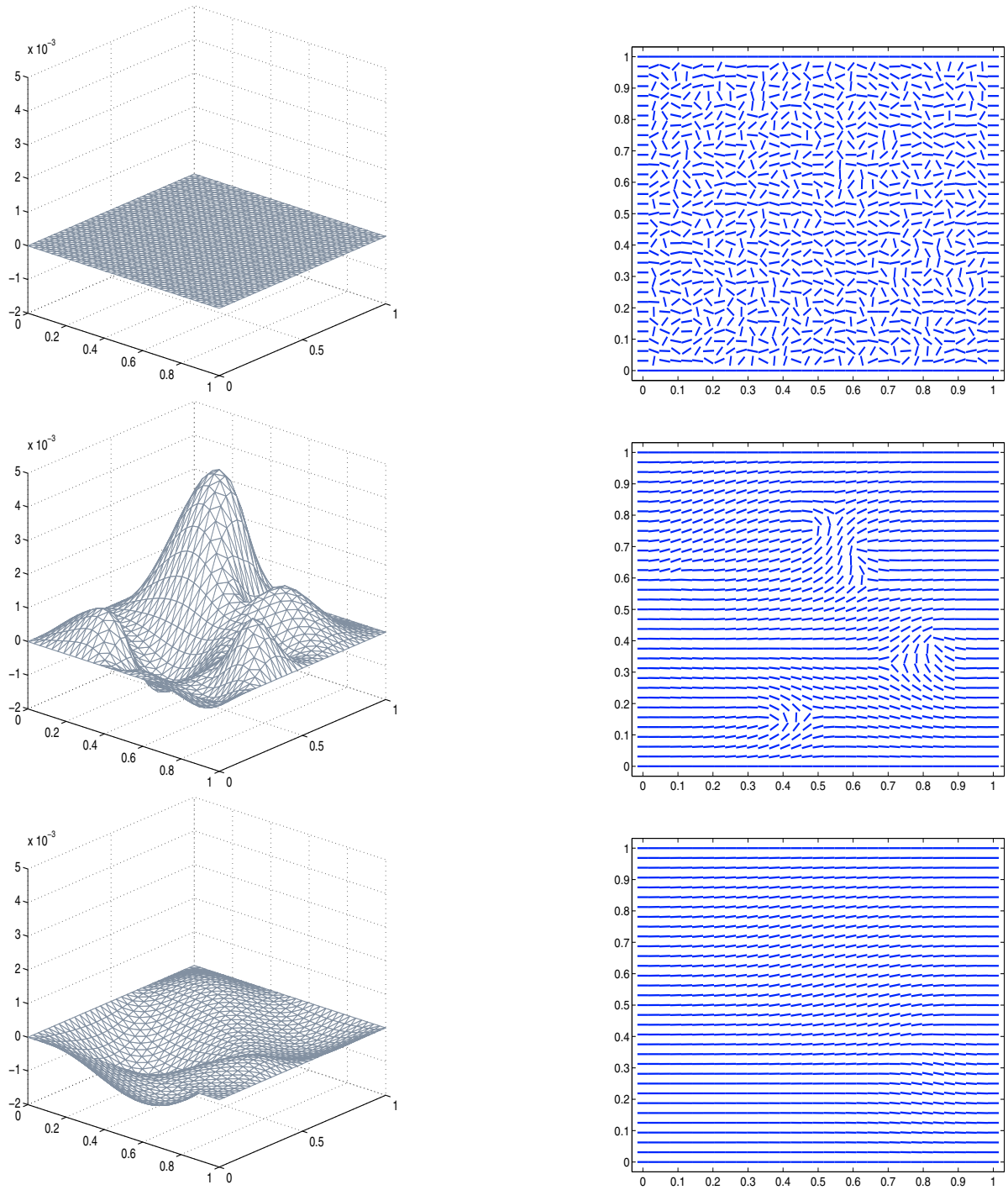

Figure 2. Graph of the displacement $u_{h}(t, \cdot)$ and the director field $n_{h}(t, \cdot)$ during the evolution for $t=0.0,0.1$, and 0.3 (from top to bottom) in Section 6.2 with random initial data of $q$. The gradient of the order parameter leads to a locally uniform orientation with a few characteristic perturbations which introduce through the coupling a deformation of the membrane. Once the director field is uniform the deformation of the membrane is relaxed. At $t=1.0$ it is of order $10^{-4}$.

\subsection{Discrete defects and their effect on shape}

We conclude the discussion of the practical performance of our algorithm with an experiment that is not entirely covered by our theory in the sense that the discrete boundary data enforce director fields and hence order parameters whose energy cannot be uniformly bounded as $h \rightarrow 0$. This effect is due to a nonvanishing winding number of the boundary data which thus cannot be extended to the entire domain by a continuous unitlength vector field. Nevertheless, the mesh-size may be regarded as a length scale comparable to the physical scale and then the discrete evolution is still meaningful though mesh-dependent. 

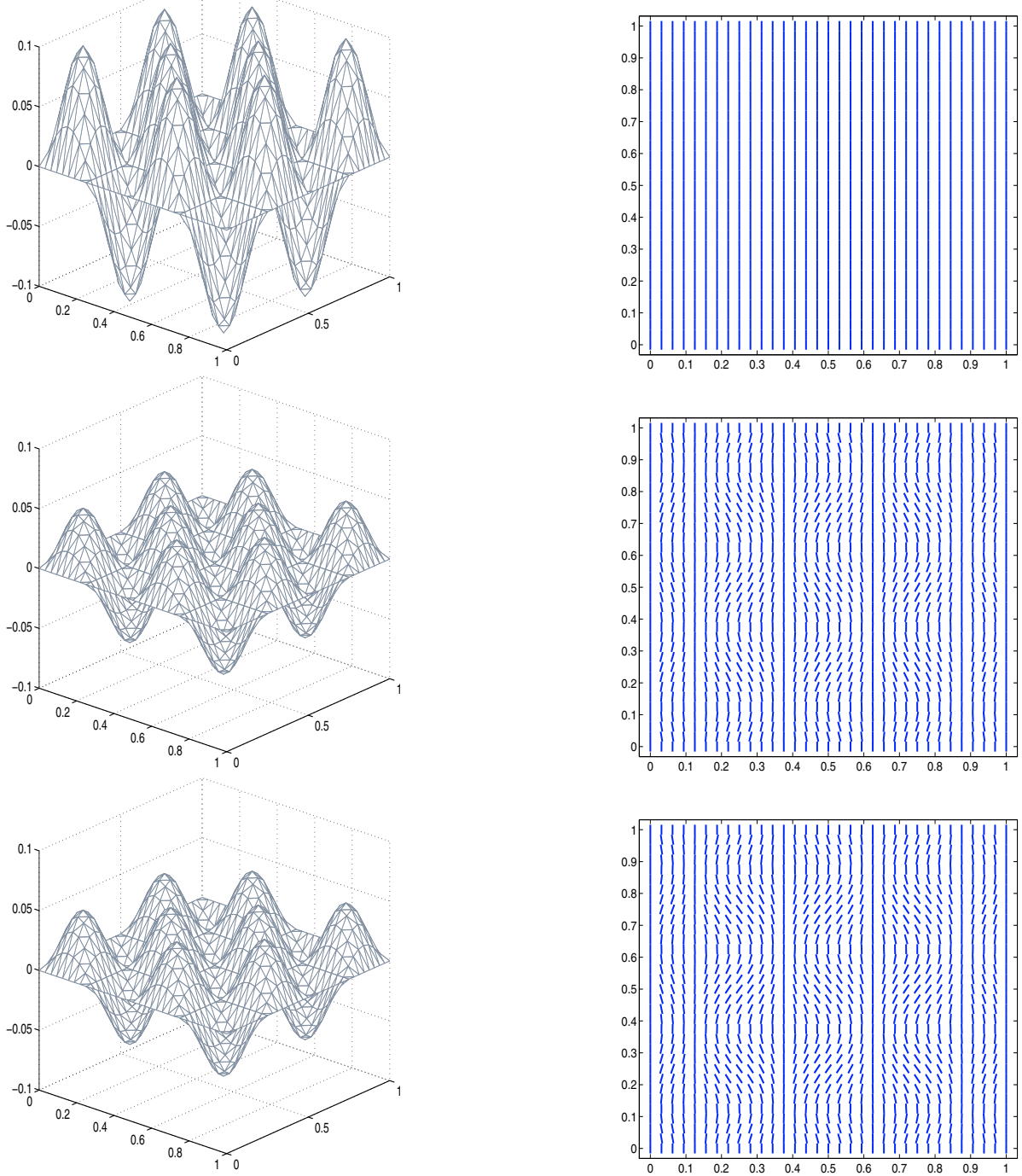

FiguRE 3. Graph of the displacement $u_{h}(t, \cdot)$ and the director field $n_{h}(t, \cdot)$ at $t=0.0,0.2,0.4$ (from top to bottom) in Section 6.3 which includes spontaneous curvature. The observed patterns are consistent with the heuristic considerations in Section 2.3. The principle curvature directions at the saddles (e.g. in the center of the sample) are parallel to $(1, \pm 1)$. We also notice the effect of the boundary conditions which dominates the behavior close to the boundary of the sample. This configuration is very stable and does not change significantly during the remaining part of the evolution up to time $t=1.0$.

In polar coordinates $\left(r_{a}, \theta_{a}\right)$ about $\left(a_{1}, a_{2}\right)=(1 / 4,1 / 2)$ and $\left(r_{b}, \theta_{b}\right)$ about $\left(b_{1}, b_{2}\right)=(3 / 4,1 / 2)$ we define

$$
n\left(x_{1}, x_{2}\right)= \begin{cases}\left(\cos \left(\theta_{a} / 2\right), \sin \left(\theta_{a} / 2\right)\right) & \text { for } x_{1} \leq 1 / 2 \\ \left(\cos \left(-\left(\theta_{b}+\pi\right)\right), \sin \left(-\left(\theta_{b}+\pi\right)\right)\right) & \text { for } x_{1}>1 / 2\end{cases}
$$

and set

$$
q_{h}^{0}=\mathcal{I}_{h}\left[\left(2 n_{1}^{2}-1 / 2,2 n_{1} n_{2}\right)\right]
$$




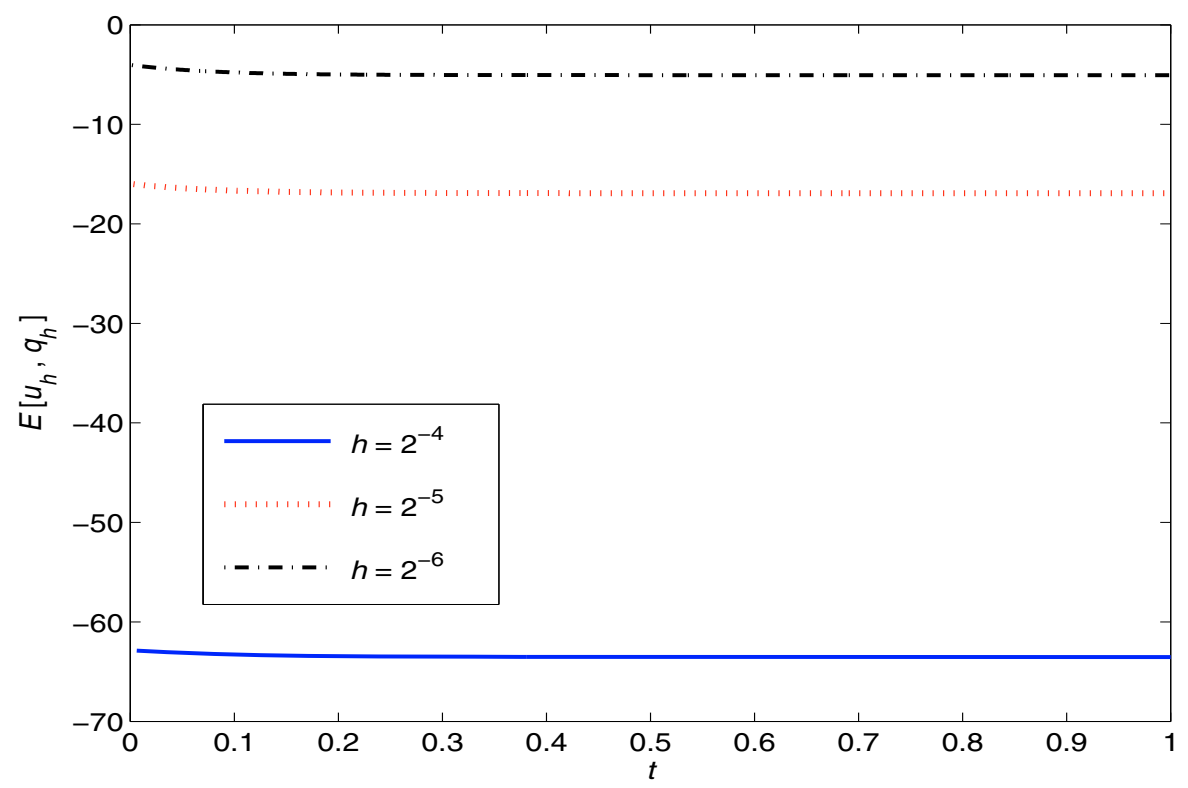

Figure 4. Discrete energy $E_{h}\left[u_{h}(t), q_{h}(t)\right]$ as a function of $t \in[0,1]$ for the setting including spontaneous curvature defined in Section 6.3.

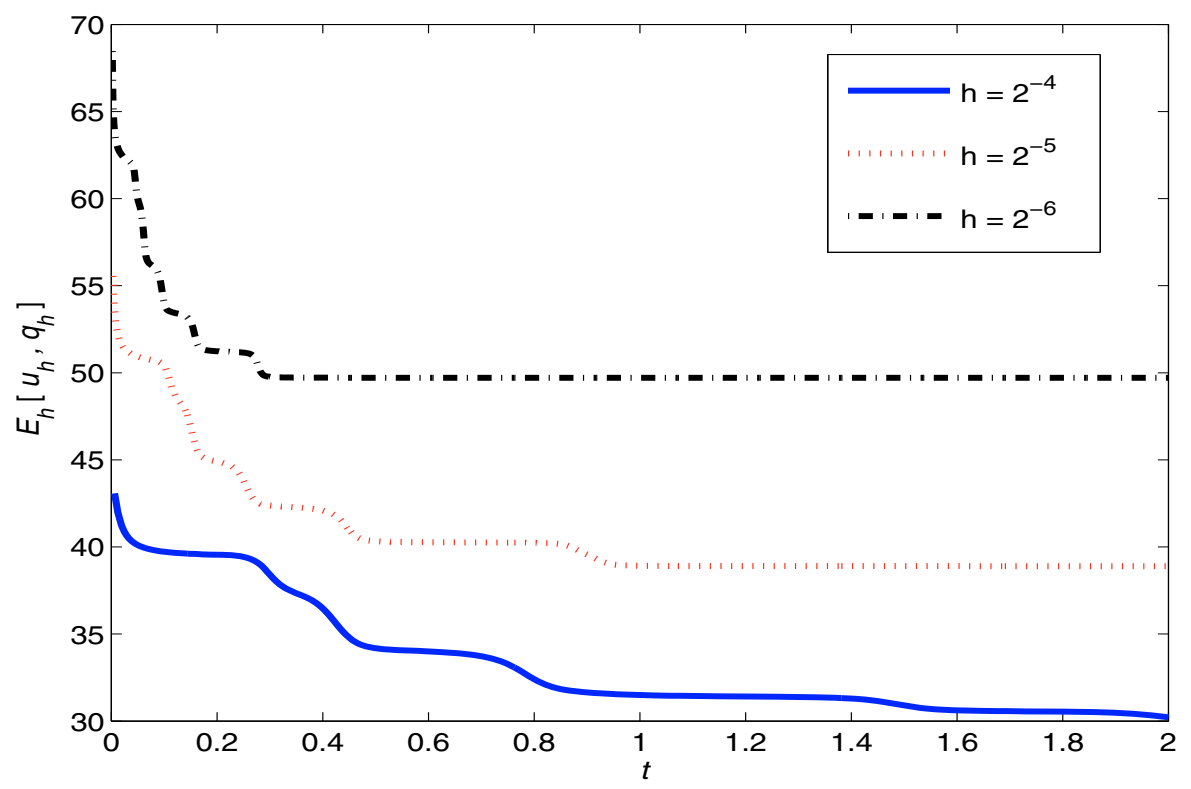

Figure 5. Discrete energy $E_{h}\left[u_{h}(t), q_{h}(t)\right]$ as a function of $t \in[0,1]$ for the initial data including a degree -1 and a degree $1 / 2$ singularity defined in Section 6.4. 

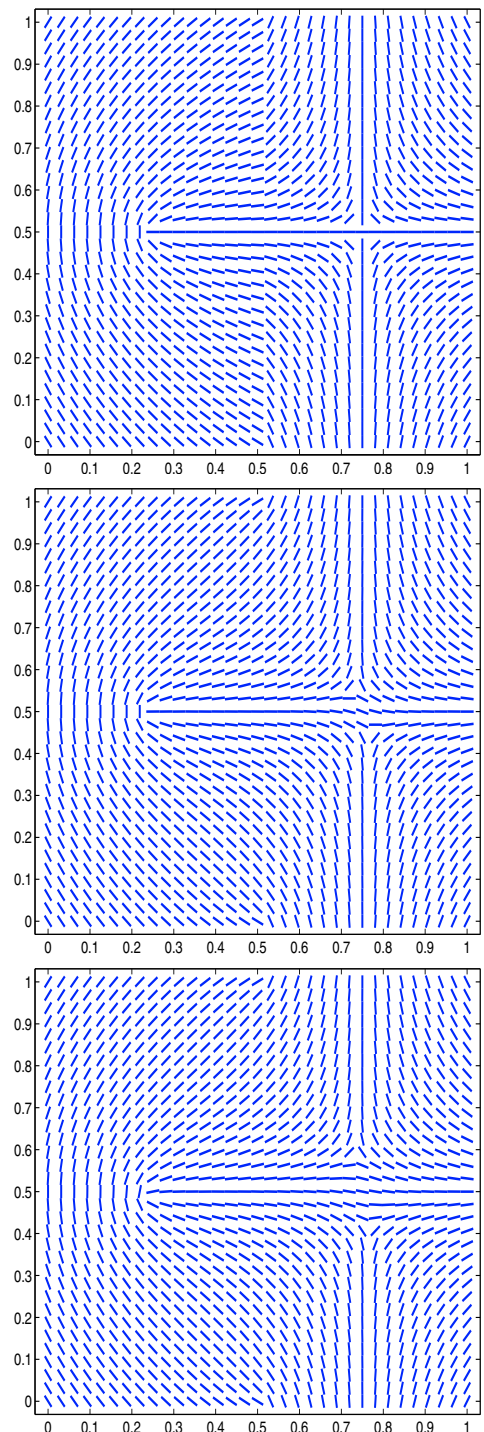
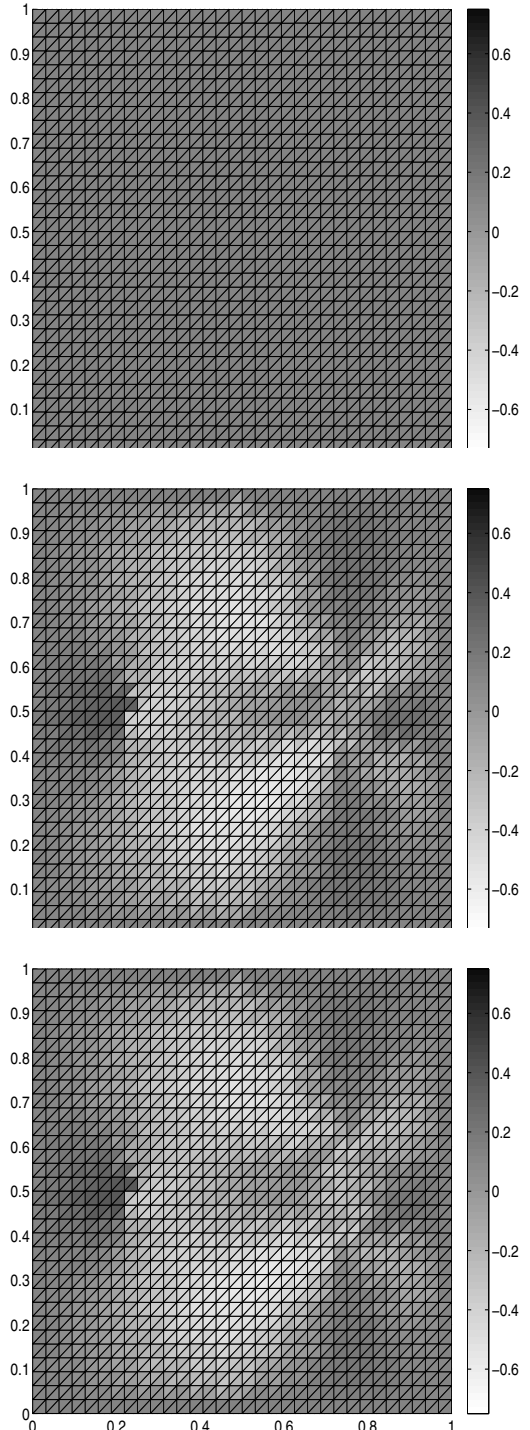

Figure 6 . The director field $n_{h}(t, \cdot)$ and the discrete Laplacian $\Delta_{h} u_{h}(t, \cdot)$ at the early stages of the evolution for $t=0.0,0.2,0.4$ in the example in Section 6.4. Note that the singularity of degree -1 located at $(3 / 4,1 / 2)$ splits immediately into two singularities of degree $-1 / 2$. The singularity of degree $1 / 2$ located at $(1 / 4,1 / 2)$ has a much stronger influence on the curvature of the surface. This configuration is very stable and does not change significantly during the remaining part of the evolution. As predicted by Uchida [41], we observe that a singularity of strength $-1 / 2$ carries three lobes of positive curvature in which the director points toward the core of the singularity. A singularity of strength $+1 / 2$ carries one lobe of positive curvature and the curvature is negative in the region in which the director points toward the core. 
together with $u_{h}^{0} \equiv 0$. Moreover, we use

$$
T=2, \quad \gamma_{q}=\gamma_{u}=0.1, \quad \kappa=1, \quad M=1, \quad \delta=1
$$

Figure 6 shows the director field and the induced curvature for various times. The degree -1 singularity initially located at $(3 / 4,1 / 2)$ immediately splits into two singularities of degree $-1 / 2$ which is energetically preferable. In fact, the Frank energy of a singularity of strength $s$ in a neighborhood of the core after removing a small disc of radius $\epsilon$ centered at the core is of order

$$
\int_{B(0,1) \backslash B(0, \epsilon)}|\nabla n|^{2} \mathrm{~d} x \sim \int_{B(0,1) \backslash B(0, \epsilon)} \frac{s^{2}}{|x|^{2}} \mathrm{~d} x \sim s^{2}|\log \epsilon| .
$$

In our discretized setting the cut-off radius is comparable to $h$ thus we expect that the splitting of the singularity of strength -1 into two singularities of strength $-1 / 2$ should be accompanied by a decay of the energy of order $|\log h| / 2$. Indeed, the numerical simulations confirm that this splitting is accompanied by a large decay of the energy and the energy decays further as the singularities separate, $c f$. Figure 5. The motion of the topological defects seems to be mesh-dependent and whenever they move by one layer in the mesh the energy is reduced to a lower level. The degree $1 / 2$ singularity seems to have a larger influence on the curvature than the ones of negative degree. It is interesting to note that the defects of opposite sign did not tend to interact on the time scale of our simulation. However, we expect that defects of degree $1 / 2$ and $-1 / 2$, respectively, will annihilate by diffusion for large times.

Acknowledgements. The work of G.D. was partially supported by the NSF through grants DMS-0104118 and DMS0405853, and the work of R.H.N. by the NSF through grants DMS-0505454 and DMS-0807811 and the Vielberth foundation. S.B. acknowledges financial support from the DAAD.

\section{REFERENCES}

[1] F. Alouges, A new algorithm for computing liquid crystal stable configurations: the harmonic mapping case. SIAM J. Numer. Anal. 34 (1997) 1708-1726.

[2] F. Alouges, A new finite element scheme for Landau-Lifchitz equations. Discrete Contin. Dyn. Syst. Ser. S 1 (2008) $187-196$.

[3] J.W. Barrett, S. Bartels, X. Feng and A. Prohl, A convergent and constraint-preserving finite element method for the p-harmonic flow into spheres. SIAM J. Numer. Anal. 45 (2007) 905-927.

[4] J.W. Barrett, H. Garcke and R. Nürnberg, On the parametric finite element approximation of evolving hypersurfaces in $\mathbb{R}^{3}$. J. Comput. Phys. 227 (2008) 4281-4307.

[5] S. Bartels, Stability and convergence of finite-element approximation schemes for harmonic maps. SIAM J. Numer. Anal. 43 (2005) 220-238 (electronic).

[6] T. Baumgart, S.T. Hess and W.W. Webb, Imaging co-existing domains in biomembrane models coupling curvature and tension. Nature 425 (2003) 832-824.

[7] T. Biben and C. Misbah, An advected-field model for deformable entities under flow. Eur. Phys. J. B 29 (2002) 311-316.

[8] P. Biscari and E.M. Terentjev, Nematic membranes: Shape instabilities of closed achiral vesicles. Phys. Rev. E 73 (2006) 051706.

[9] F. Brezzi and M. Fortin, Mixed and hybrid finite element methods, Springer Series in Computational Mathematics 15. SpringerVerlag, New York, USA (1991).

[10] P.B. Canham, The minimum energy of bending as a possible explanation of the biconcave shape of the human red blood cell. J. Theort. Biol. 26 (1970) 61-81.

[11] Y.M. Chen, The weak solutions to the evolution problems of harmonic maps. Math. Z. 201 (1989) 69-74.

[12] C.H.A. Cheng, D. Coutand and S. Shkoller, Navier-Stokes equations interacting with a nonlinear elastic biofluid shell. SIAM J. Math. Anal. 39 (2007) 742-800 (electronic).

[13] P.G. Ciarlet, The finite element method for elliptic problems, Classics in Applied Mathematics 40. Society for Industrial and Applied Mathematics (SIAM), Philadelphia, USA (2002). Reprint of the 1978 original [North-Holland, Amsterdam; MR0520174 (58 \#25001)].

[14] K. Deckelnick, G. Dziuk and C.M. Elliott, Computation of geometric partial differential equations and mean curvature flow. Acta Numer. 14 (2005) 139-232. 
[15] Q. Du, C. Liu and X. Wang, A phase field approach in the numerical study of the elastic bending energy for vesicle membranes. J. Comput. Phys. 198 (2004) 450-468.

[16] Q. Du, C. Liu and X. Wang, Simulating the deformation of vesicle membranes under elastic bending energy in three dimensions. J. Comput. Phys. 212 (2006) 757-777.

[17] G. Dziuk, Computational parametric Willmore flow. Numer. Math. 111 (2008) 55-80.

[18] E. Evans, Bending resistance and chemically induced moments in membrane bilayers. Biophys. J. 14 (1974) $923-931$.

[19] J.B. Fournier and P. Galatoa, Sponges, tubules and modulated phases of para-antinematic membranes. J. Phys. II 7 (1997) 1509-1520.

[20] A. Freire, S. Müller and M. Struwe, Weak compactness of wave maps and harmonic maps. Ann. Inst. H. Poincaré Anal. Non Linéaire 15 (1998) 725-754.

[21] M. Giaquinta and S. Hildebrandt, Calculus of variations I: The Lagrangian formalism, Grundlehren der Mathematischen Wissenschaften 310, [Fundamental Principles of Mathematical Sciences]. Springer-Verlag, Berlin, Germany (1996).

[22] P. Grisvard, Elliptic problems in nonsmooth domains, Monographs and Studies in Mathematics 24. Pitman (Advanced Publishing Program), Boston, USA (1985).

[23] C. Grossmann and H.-G. Roos, Numerical treatment of partial differential equations. Universitext, Springer, Berlin, Germany (2007). Translated and revised from the 3rd (2005) German edition by Martin Stynes.

[24] W. Helfrich, Elastic properties of lipid bilayers: theory and possible experiments. Z. Naturforsch. C 28 (1973) $693-703$.

[25] W. Helfrich and J. Prost, Intrinsic bending force in anisotropic membranes made of chiral molecules. Phys. Rev. A 38 (1988) 3065-3068.

[26] J.T. Jenkins, The equations of mechanical equilibrium of a model membrane. SIAM J. Appl. Math. 32 (1977) $755-764$.

[27] M.A. Johnson and R.S. Decca, Dynamics of topological defects in the $l_{\beta^{\prime}}$ phase of 1 , 2-dipalmitoyl phosphatidylcholine bilayers. Opt. Commun. 281 (2008) 1870-1875.

[28] O.A. Ladyzhenskaya and N.N. Ural'tseva, Linear and quasilinear elliptic equations. Academic Press, New York, USA (1968). Translated from the Russian by Scripta Technica, Inc. Translation editor: Leon Ehrenpreis.

[29] T. C. Lubensky and F.C. MacKintosh, Theory of "ripple" phases of bilayers. Phys. Rev. Lett. 71 (1993) $1565-1568$.

[30] F.C. MacKintosh and T.C. Lubensky, Orientational order, topology, and vesicle shapes. Phys. Rev. Lett. 67 (1991) $1169-1172$.

[31] S.J. Marrink, J. Risselada and A.E. Mark, Simulation of gel phase formation and melting in lipid bilayers using a coarse grained model. Chem. Phys. Lipids 135 (2005) 223-244.

[32] S.T.-N.J.F. Nagle, Structure of lipid bilayers. Biochim. Biophys. Acta 1469 (2000) 159-195.

[33] P. Nelson and T. Powers, Rigid chiral membranes. Phys. Rev. Lett. 69 (1992) 3409-3412.

[34] R. Oda, I. Huc, M. Schmutz and S.J. Candau, Tuning bilayer twist using chiral counterions. Nature 399 (1999) 566-569.

[35] M.S. Pauletti, Parametric AFEM for geometric evolution equations coupled fluid-membrane interaction. Ph.D. Thesis, University of Maryland, USA (2008).

[36] R.E. Rusu, An algorithm for the elastic flow of surfaces. Interfaces Free Bound. 7 (2005) $229-239$.

[37] U. Seifert, Configurations of fluid membranes and vesicles. Adv. Phys. 46 (1997) 13-137.

[38] J.V. Selinger and J.M. Schnur, Theory of chiral lipid tubules. Phys. Rev. Lett. 71 (1993) 4091-4094.

[39] D. Steigmann, Fluid films with curvature elasticity. Arch. Ration. Mech. Anal. 150 (1999) $127-152$.

[40] M. Struwe, Geometric evolution problems, in Nonlinear partial differential equations in differential geometry (Park City, UT, 1992), IAS/Park City Math. Ser. 2, Amer. Math. Soc., Providence, USA (1996) 257-339.

[41] N. Uchida, Dynamics of orientational ordering in fluid membranes. Phys. Rev. E 66 (2002) 040902.

[42] E.G. Virga, Variational theories for liquid crystals, Appl. Math. Math. Comput. 8. Chapman \& Hall, London, UK (1994).

[43] T.J. Willmore, Riemannian geometry, Oxford Science Publications. The Clarendon Press Oxford University Press, New York, USA (1993). 